\title{
FINITE ELEMENT APPROXIMATION OF THE THREE-FIELD FORMULATION OF THE STOKES PROBLEM USING ARBITRARY INTERPOLATIONS*
}

\author{
RAMON CODINA ${ }^{\dagger}$
}

\begin{abstract}
The stress-displacement-pressure formulation of the elasticity problem may suffer from two types of numerical instabilities related to the finite element interpolation of the unknowns. The first is the classical pressure instability that occurs when the solid is incompressible, whereas the second is the lack of stability in the stresses. To overcome these instabilities, there are two options. The first is to use different interpolation for all the unknowns satisfying two inf-sup conditions. Whereas there are several displacement-pressure interpolations that render the pressure stable, less possibilities are known for the stress interpolation. The second option is to use a stabilized finite element formulation instead of the plain Galerkin approach. If this formulation is properly designed, it is possible to use arbitrary interpolation for all the unknowns. The purpose of this paper is precisely to present one of such formulations. In particular, it is based on the decomposition of the unknowns into their finite element component and a subscale, which will be approximated and whose goal is to yield a stable formulation. A singular feature of the method to be presented is that the subscales will be considered orthogonal to the finite element space. We describe the design of the formulation and present the results of its numerical analysis.
\end{abstract}

Key words. Stokes problem, stress-displacement-pressure, stabilized finite elements

AMS subject classifications. 65N12, 65N30, 76D07

DOI. $10.1137 / 080712726$

1. Introduction. The analysis of the three-field formulation of the linear elastic incompressible problem is probably not a goal by itself, but rather a simple model to study problems in which it is important to interpolate the stresses independently from the displacements and, in the case we will consider, also the pressure. Perhaps the most salient problem that requires the interpolation of the (deviatoric) stresses is the viscoelastic one. In this case, the algebraic constitutive equation (linear or nonlinear) that relates stresses and strains has to be replaced by an evolution equation (see [3] for a review).

The problem we will study in this paper is the simple Stokes problem arising in linear elasticity or creeping flows, taking as unknowns the displacement field (or velocity field, in a fluid problem), the pressure, and the deviatoric part of the stresses. In particular, we shall consider that the material is incompressible.

When the finite element approximation of the problem is undertaken, it is well known that incompressibility poses a stringent requirement in the way the pressure is interpolated with respect to the displacement field. The displacement and pressure finite element spaces have to satisfy the classical inf-sup condition [8]. Several interpolations are known that satisfy this condition and yield a stable displacementpressure numerical solution. However, less is known about another inf-sup condition that needs to be satisfied when the stresses are interpolated independently from the displacement. This inf-sup condition is trivially satisfied for the continuous problem, but only a few interpolations are known that verify it for the discrete case. It is

\footnotetext{
${ }^{*}$ Received by the editors January 8, 2008; accepted for publication (in revised form) September 17, 2008; published electronically January 16, 2009.

http://www.siam.org/journals/sinum/47-1/71272.html

†Department de Resistencia de Materias i Estructures a l'Enginyeria, Universitat Politècnica de Catalunya, Jordi Girona 1-3, Edifici C1, 08034 Barcelona, Spain (ramon.codina@upc.edu).
}

699 
discussed, for example, in [25]. In the context of viscoelastic flows, a popular stable three-field interpolation was introduced in [23], and the numerical analysis was undertaken in [15]. See also $[28,26]$ for other contributions proposing different stable finite element interpolations.

The inf-sup conditions for the displacement-pressure and stresses-displacement interpolations are needed if the standard Galerkin method is used for the space discretization. However, there is also the possibility to resort to a stabilized finite element method, in which the discrete variational form of the Galerkin formulation is modified in order to enhance its stability. The purpose of this paper is precisely to present one of such formulations. In particular, the one proposed here is based on the decomposition of the unknowns into their finite element component and a subscale, that is, the component of the continuous unknown that cannot be captured by the finite element mesh. Obviously, this subscale needs to be approximated in one way or another. This idea was proposed in the finite element context in $[20,21]$ and termed variational multiscale approximation, although there are similar concepts developed in different situations (both in physical and numerical modeling).

The important property of the formulation to be presented here is that the subscale will be considered orthogonal to the appropriate finite element space. This idea was first applied to the Stokes problem in displacement-pressure form in [9], and subsequently applied to general incompressible flows in [10]. Likewise, we will introduce a way to motivate an expression for the subscales on the element boundaries. These will allow us to consider stress, or both. We will I displacement interpolati Other stabilization gradient to deal with the simple method based on projectin
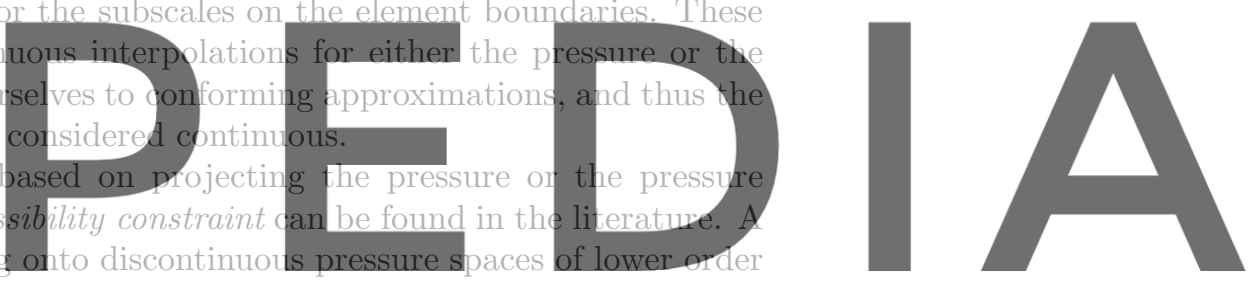
can be found in [13]. In [4] a method based on projecting onto pressures defined

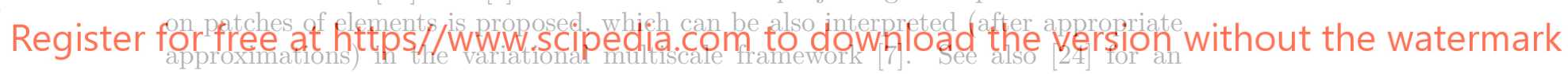
abstract analysis and generalization of these type of methods. Nevertheless, some conditions on the finite element mesh are often required that are difficult to meet in practical unstructured finite element meshes.

Different stabilized formulations for the three-field Stokes problem can be found in the literature. The GLS (Galerkin/least-squares) method is used, for example, in $[5,16,27]$. In $[19,14]$ the authors propose what they call EVSS (elastic-viscous-splitstress), which is related to the formulation proposed in this paper in what concerns the way to stabilize the stress interpolation. An analysis of both approaches, GLS and EVSS, is presented in [6].

Even though our interest is to consider incompressible materials and therefore to include the pressure as a variable, a similar formulation to the one proposed here could be applied to other versions of the elasticity problem. The difficulty to devise stable total stress-displacement interpolations is well known (see, for example, [2] and also the general approach adopted in [1]). A stabilized formulation for the stressdisplacement-rotation formulation can be found in [17] (in 2D) and [18] (in 3D). In these references the stability of the Galerkin formulation is also enhanced by adding some least-square-type terms. The application of the formulation to be presented to different versions of the elasticity problem would be straightforward.

The paper is organized as follows. In the following section we present the problem to be solved and its Galerkin finite element approximation, explaining the sources of 
numerical instability. Then we present the stabilized finite element formulation we propose, for which we present a complete numerical analysis in section 4 . The paper concludes with some final remarks.

\section{Problem statement and Galerkin finite element discretization.}

2.1. Boundary value problem. Let $\Omega$ be the computational domain of $\mathbb{R}^{d}$ ( $d=2$ or 3 ) occupied by the solid (or fluid), assumed to be bounded and polyhedral, and let $\partial \Omega$ be its boundary. If $\boldsymbol{u}$ is the displacement field, $p$ the pressure (taken as positive in compression), and $\boldsymbol{\sigma}$ the deviatoric component of the stress field, the field equations to be solved in the domain $\Omega$ are

$$
\begin{array}{r}
-\nabla \cdot \boldsymbol{\sigma}+\nabla p=\boldsymbol{f}, \\
\nabla \cdot u=0, \\
\frac{1}{2 \mu} \sigma-\nabla^{S} u=0,
\end{array}
$$

where $\boldsymbol{f}$ is the vector of body forces, $\mu$ the shear modulus, and $\nabla^{S} \boldsymbol{u}$ the symmetrical part of $\nabla \boldsymbol{u}$. For simplicity, we shall consider the simplest boundary condition $u=0$ on $\partial \Omega$.

2.2. Variational form. To write the weak form of problem (2.1)-(2.3) we
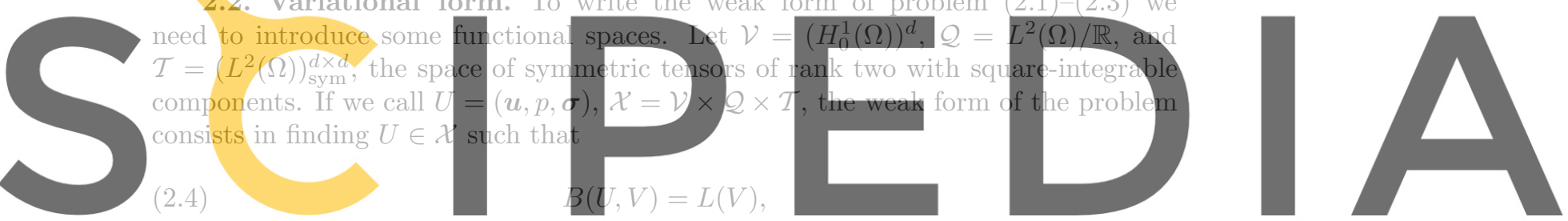

for all $V=(v, q, \tau) \in \mathcal{X}$, where

Register for free at https//www.scipedia.com to download the version without the watermark

$$
\begin{aligned}
B(U, V) & =\left(\nabla^{S} v, \sigma\right)-(p, \nabla \cdot v)+(q, \nabla \cdot u)+\frac{1}{2 \mu}(\sigma, \tau)-\left(\nabla^{S} u, \tau\right), \\
L(V) & =\langle\boldsymbol{f}, \boldsymbol{v}\rangle,
\end{aligned}
$$

where $(\cdot, \cdot)$ is the $L^{2}$ inner product and $\langle\cdot, \cdot\rangle$ is the duality pairing between $\mathcal{V}$ and its dual, $\left(H^{-1}(\Omega)\right)^{d}$, where $\boldsymbol{f}$ is assumed to belong.

2.3. Stability of the Galerkin finite element discretization. Let us consider a finite element partition $\mathcal{P}_{h}$ of the domain $\Omega$ of diameter $h$. For simplicity, we will consider quasi-uniform refinements, and thus all the element diameters can be bounded above and below by constants multiplying $h$. The extension of the following analysis to general shape-regular meshes (also called nondegenerate meshes) can be done using the strategy developed in [11].

From the finite element partition we may build up conforming finite element spaces $\mathcal{V}_{h} \subset \mathcal{V}, \mathcal{Q}_{h} \subset \mathcal{Q}$, and $\mathcal{T}_{h} \subset \mathcal{T}$ in the usual manner. If $\mathcal{X}_{h}=\mathcal{V}_{h} \times \mathcal{Q}_{h} \times \mathcal{T}_{h}$ and $U_{h}=\left(\boldsymbol{u}_{h}, p_{h}, \boldsymbol{\sigma}_{h}\right)$, the Galerkin finite element approximation consists in finding $U_{h} \in \mathcal{X}_{h}$ such that

$$
B\left(U_{h}, V_{h}\right)=L\left(V_{h}\right)
$$

for all $V_{h}=\left(\boldsymbol{v}_{h}, q_{h}, \boldsymbol{\tau}_{h}\right) \in \mathcal{X}_{h}$. 
In principle, we have posed no restrictions on the choice of the finite element spaces. However, let us analyze the numerical stability of problem (2.7). If we take $V_{h}=U_{h}$, it is found that

$$
B\left(U_{h}, U_{h}\right)=\frac{1}{2 \mu}\left\|\boldsymbol{\sigma}_{h}\right\|^{2},
$$

where $\|\cdot\|$ is the $L^{2}(\Omega)$ norm. It is seen from (2.8) that $B_{h}$ is not coercive in $\mathcal{X}_{h}$, the displacement and the pressure being out of control. Moreover, the inf-sup condition

$$
\inf _{U_{h} \in \mathcal{X}_{h}} \sup _{V_{h} \in \mathcal{X}_{h}} \frac{B\left(U_{h}, V_{h}\right)}{\left\|U_{h}\right\| \mathcal{X}\left\|V_{h}\right\|_{\mathcal{X}}} \geq \beta
$$

is not satisfied for any positive constant $\beta$ unless the two conditions

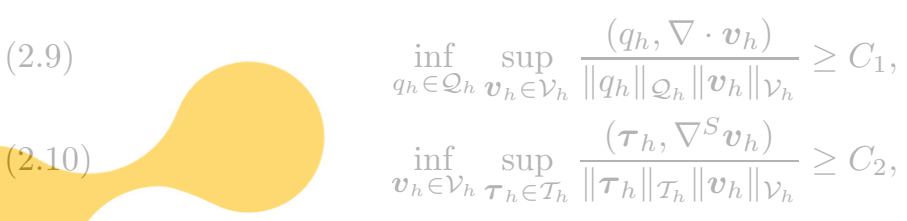

hold for positive constants $C_{1}$ and $C_{2}$ (see, for example, [25]). In all the expressions above, $\|\cdot\|_{\mathcal{Y}}$ stands for the appropriate norm in space $\mathcal{Y}$.

Conditions (2.9) and (2.10) pose stringent requirements on the choice of the finite element spaces. Our intention in this paper is to present a stabilized finite element formulation that avoids interpolation for all the and we will assume that lations of degree $k_{u}, k_{p}$ but the stress and press
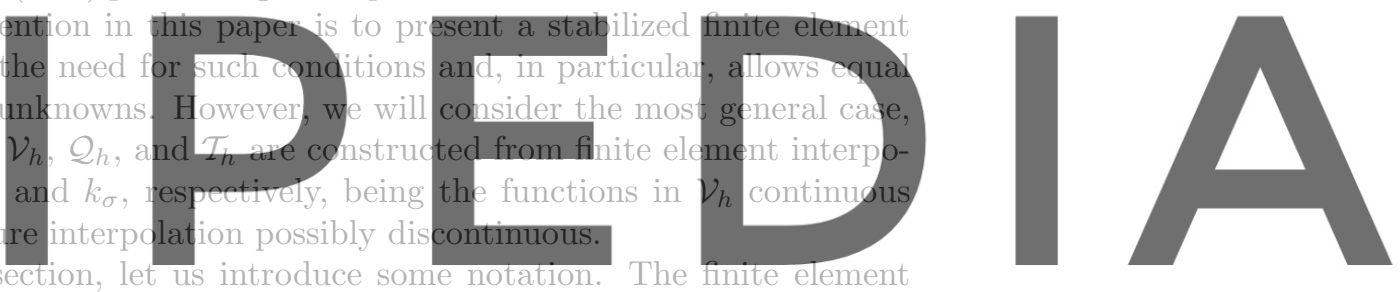

partition will be denoted by $\mathcal{P}_{h}=\{K\}$, and summation over all the elements will

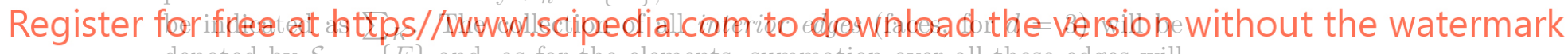
denoted by $\mathcal{E}_{h}=\{E\}$ and, as for the elements, summation over all these edges will be indicated as $\sum_{E}$. The symbol $\langle f, g\rangle_{D}$ will be used to denote the integral of the product of functions $f$ and $g$ over $D$, with $D=K$ (an element), $D=\partial K$ (an element boundary), or $D=E$ (an edge). Likewise, $\|f\|_{D}^{2}:=\langle f, f\rangle_{D}$. Suppose now that elements $K_{1}$ and $K_{2}$ share an edge $E$, and let $\boldsymbol{n}_{1}$ and $\boldsymbol{n}_{2}$ be the normals to $E$ exterior to $K_{1}$ and $K_{2}$, respectively. For a scalar function $f$, possibly discontinuous across $E$, we define its jump as $\llbracket \boldsymbol{n} f \rrbracket_{E}:=\left.\boldsymbol{n}_{1} f\right|_{\partial K_{1} \cap E}+\left.\boldsymbol{n}_{2} f\right|_{\partial K_{2} \cap E}$, and for a vector or tensor $\boldsymbol{v}, \llbracket \boldsymbol{n} \cdot \boldsymbol{v} \rrbracket_{E}:=\left.\boldsymbol{n}_{1} \cdot \boldsymbol{v}\right|_{\partial K_{1} \cap E}+\left.\boldsymbol{n}_{2} \cdot \boldsymbol{v}\right|_{\partial K_{2} \cap E}$.

3. Design of the stabilized finite element approximation using subscales. In this section we describe the finite element formulation proposed. The arguments in this design step are necessarily heuristic. Their validity depends on the numerical performance of the formulation, which will not be checked here (see the final remarks in section 5), and on the numerical analysis to be presented in the following section.

3.1. Decomposition of the unknowns. Let us start by explaining the basic idea of the multiscale formulation proposed in [20] and applying it to our problem. If we split $U=U_{h}+U^{\prime}$, where $U_{h}$ belongs to the finite element space $\mathcal{X}_{h}$ and $U^{\prime}$ to any space $\mathcal{X}^{\prime}$ to complement $\mathcal{X}_{h}$ in $\mathcal{X}$, problem (2.4) is exactly equivalent to

$$
B\left(U_{h}+U^{\prime}, V_{h}\right)=L\left(V_{h}\right) \quad \forall V_{h} \in \mathcal{X}_{h},
$$




$$
B\left(U_{h}+U^{\prime}, V^{\prime}\right)=L\left(V^{\prime}\right) \quad \forall V^{\prime} \in \mathcal{X}^{\prime} .
$$

In essence, the goal of all subscale methods, including the approximation with bubble functions, is to approximate $U^{\prime}$ in one way or another and end up with a problem for $U_{h}$ alone.

Integrating some terms by parts and using the fact that $\boldsymbol{u}_{h}=\boldsymbol{u}^{\prime}=\mathbf{0}$ on $\partial \Omega$, it is easy to see that (3.1) in our case can be written as

$$
\begin{aligned}
& B\left(U_{h}, V_{h}\right)+\left(\nabla^{S} \boldsymbol{v}_{h}, \boldsymbol{\sigma}^{\prime}\right)-\left(p^{\prime}, \nabla \cdot \boldsymbol{v}_{h}\right)+\frac{1}{2 \mu}\left(\boldsymbol{\sigma}^{\prime}, \boldsymbol{\tau}_{h}\right) \\
& \quad+\sum_{E}\left\langle\boldsymbol{u}_{E}^{\prime}, \llbracket \boldsymbol{n} q_{h}-\boldsymbol{n} \cdot \boldsymbol{\tau}_{h} \rrbracket\right\rangle_{E}+\sum_{K}\left\langle\boldsymbol{u}_{K}^{\prime},-\nabla q_{h}+\nabla \cdot \boldsymbol{\tau}_{h}\right\rangle_{K}=L\left(V_{h}\right),
\end{aligned}
$$

where we have distinguished between the displacement subscale in the elements interiors, $\boldsymbol{u}_{K}^{\prime}$, and on the edges, $\boldsymbol{u}_{E}^{\prime}$. The stress and pressure subscales are required only in the element interiors (recall that they may be discontinuous).

On the other hand, integrating back some terms by parts in (3.2) it is found that

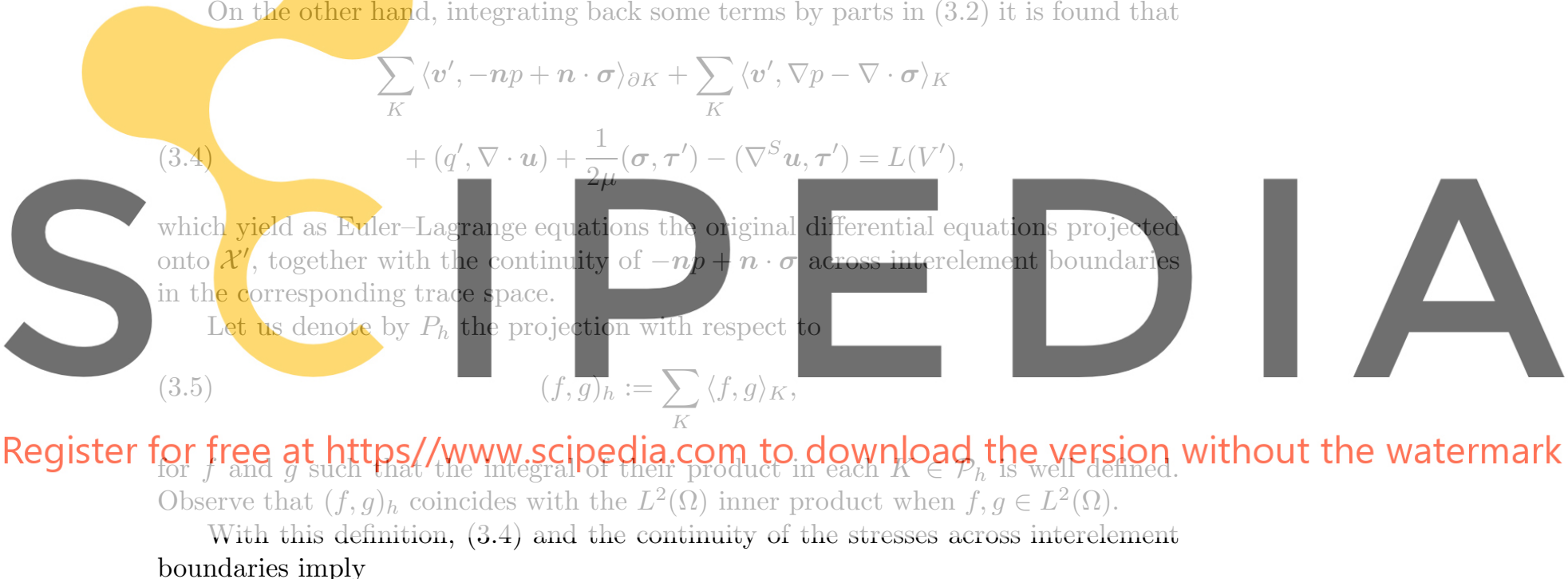

boundaries imply

$$
\left.\begin{array}{rll}
-\nabla \cdot \boldsymbol{\sigma}^{\prime}+\nabla p^{\prime}=\boldsymbol{r}_{u} & :=\boldsymbol{f}+\nabla \cdot \boldsymbol{\sigma}_{h}-\nabla p_{h}+\boldsymbol{\xi}_{u} \\
\nabla \cdot \boldsymbol{u}_{K}^{\prime}=r_{p} & :=-\nabla \cdot \boldsymbol{u}_{h}+\xi_{p} & \\
\frac{1}{2 \mu} \boldsymbol{\sigma}^{\prime}-\nabla^{S} \boldsymbol{u}_{K}^{\prime}=\boldsymbol{r}_{\sigma} & :=-\frac{1}{2 \mu} \boldsymbol{\sigma}_{h}+\nabla^{S} \boldsymbol{u}_{h}+\boldsymbol{\xi}_{\sigma}
\end{array}\right\} \begin{aligned}
& \text { in each } K \in \mathcal{P}_{h} \\
&\left.\begin{array}{rl}
\boldsymbol{u}^{\prime}=\boldsymbol{u}_{E}^{\prime} & \\
\llbracket \boldsymbol{n} p-\boldsymbol{n} \cdot \boldsymbol{\sigma} \rrbracket_{E} & =\mathbf{0}
\end{array}\right\} \text { on each } E \in \mathcal{E}_{h},
\end{aligned}
$$

where $\boldsymbol{\xi}_{u}, \xi_{p}$, and $\boldsymbol{\xi}_{\sigma}$ are orthogonal to $\mathcal{V}^{\prime}, \mathcal{Q}^{\prime}$, and $\mathcal{T}^{\prime}$, respectively, with respect to projection $P_{h}$. These vectors are responsible to enforce that the previous equations hold in the space for the subscales, which still needs to be approximated (see [10] for more details). Clearly, if (3.6) is to be understood in a classical sense, $\boldsymbol{f}$ should be more regular than required up to now and, likewise, the subscales need to be more regular than required. Nevertheless, for the moment we may assume as much regularity as needed. We will see that the final problem (3.18)-(3.19) is well defined in the functional framework introduced earlier.

The way to approximate the solution of problems (3.6)-(3.7) and to choose the space for the subscales is the topic of the following subsection. The objective is to 
obtain a closed form expression for $\boldsymbol{\sigma}^{\prime}, p^{\prime}$, and $\boldsymbol{u}_{K}^{\prime}$ defined on the element interiors and for $\boldsymbol{u}_{E}^{\prime}$ defined on the interior edges. Without any further simplification, the problem is as complex as the original one. The essential approximation step consists of approximating (3.6) without taking into account $\boldsymbol{u}_{E}^{\prime}$ and then approximating this unknown assuming the subscales on the element interiors are known.

3.2. Approximation of the subscales in the element interiors. There are several possibilities to deal with problem (3.6). As in [10], we will approximate $\boldsymbol{\sigma}^{\prime}, p^{\prime}$, and $\boldsymbol{u}^{\prime}$ by using an (approximate) Fourier analysis of the problem. We start explaining the basic idea and then we apply it to problem (3.6).

Let us consider a linear differential equation of the form $\mathcal{L} U=F$ posed in each element domain $K$, where $U$ is in general a vector unknown corresponding to a subscale, $\mathcal{L}$ a linear differential operator, and $F$ a given vector function. Let us denote the

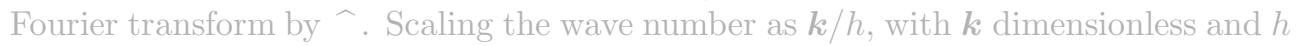
being the diameter of $K$, the basic heuristic assumption is to assume that $U$ is highly fluctuating, and thus dominated by high wave numbers. Thus, the boundary term in the Fourier transform of the derivatives can be considered negligible compared with the term involving the integral in $K$, since the former is $\mathcal{O}(1)$ and the latter $\mathcal{O}(|\boldsymbol{k}|)$. This essential approximation amounts to evaluating the Fourier transform of the equation as for functions vanishing on $\partial K$ (and extended to $\mathbb{R}^{d}$ by zero).

Suppose now that the differential equations are written in such a way that the $\longrightarrow$ product $F^{t} U$ is dimensic have the same dimension. a subscript, is an elemen is an element in the rang not be dimensionally we dimensionally consistent.
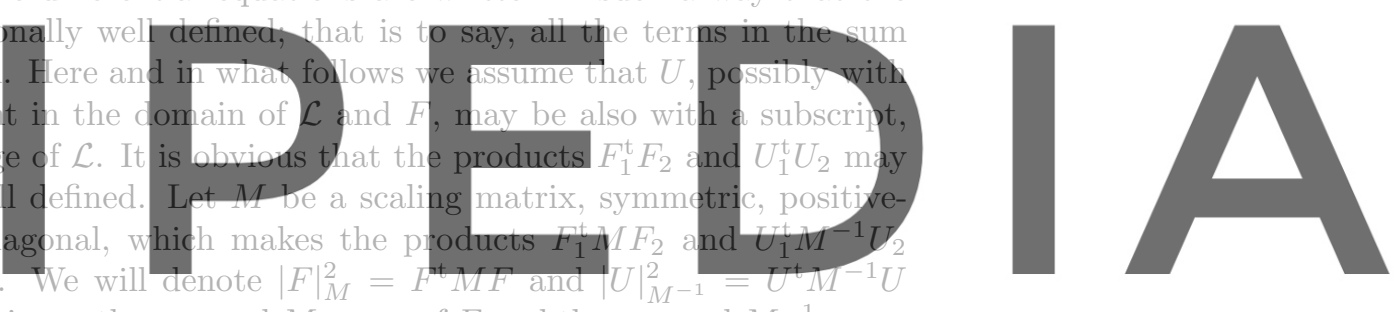
and refer to these quantities as the squared $M$-norm of $F$ and the squared $M^{-1}$-norm

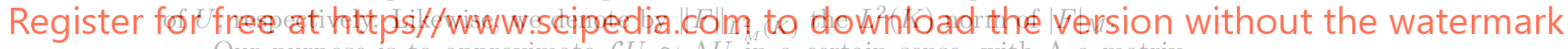

Our purpose is to approximate $\mathcal{L} U \approx \Lambda U$ in a certain sense, with $\Lambda$ a matrix

which has to be determined and that will be called matrix of stabilization parameters.

We propose to do this imposing that the induced $L_{M}^{2}(K)$ norm of $\Lambda$ is an upper bound for the induced $L_{M}^{2}(K)$ norm of $\mathcal{L}$; that is to say, $\|\mathcal{L}\|_{L_{M}^{2}(K)} \leq\|\Lambda\|_{L_{M}^{2}(K)}$. The symbol $\leq$ has to be understood up to constants and holding independently of the equation coefficients.

According to the approximation explained, we may write the Fourier transform of $\mathcal{L} U$ as $\widehat{\mathcal{L}}(\boldsymbol{k}) \widehat{U}(\boldsymbol{k})$, where $\widehat{\mathcal{L}}(\boldsymbol{k})$ is an algebraic operator. The approximate upper bound of $\|\mathcal{L}\|_{L_{M}^{2}(K)}$ can be obtained as follows. For any $U$ in the domain of $\mathcal{L}$ we have

$$
\begin{aligned}
\|\mathcal{L} U\|_{L_{M}^{2}(K)}^{2} & =\int_{K}|\mathcal{L} U|_{M}^{2} \mathrm{~d} \boldsymbol{x} \\
& \approx \int_{\mathbb{R}^{d}}|\widehat{\mathcal{L}}(\boldsymbol{k}) \widehat{U}(\boldsymbol{k})|_{M}^{2} \mathrm{~d} \boldsymbol{k} \\
& \leq \int_{\mathbb{R}^{d}}|\widehat{\mathcal{L}}(\boldsymbol{k})|_{M}^{2}|\widehat{U}(\boldsymbol{k})|_{M}^{2} \mathrm{~d} \boldsymbol{k} \\
& =\left|\widehat{\mathcal{L}}\left(\boldsymbol{k}^{0}\right)\right|_{M}^{2} \int_{\mathbb{R}^{d}}|\widehat{U}(\boldsymbol{k})|_{M}^{2} \mathrm{~d} \boldsymbol{k} \\
& \approx\left|\widehat{\mathcal{L}}\left(\boldsymbol{k}^{0}\right)\right|_{M}^{2}\|U\|_{L_{M}^{2}(K)}^{2}
\end{aligned}
$$


In the first and in the last steps we have used Plancherel's formula for the approximate Fourier transform, whereas $\boldsymbol{k}^{0}$ is a wave number whose existence is guaranteed by the mean value theorem. From the previous result it follows that $\|\mathcal{L}\|_{L_{M}^{2}(K)} \leq\left|\widehat{\mathcal{L}}\left(\boldsymbol{k}^{0}\right)\right|_{M}$ for a certain wave number, still denoted $\boldsymbol{k}^{0}$. Therefore, our proposal is to choose $\Lambda$ such that $\left|\widehat{\mathcal{L}}\left(\boldsymbol{k}^{0}\right)\right|_{M}=|\Lambda|_{M}$. Obviously, the value $\boldsymbol{k}^{0}$ is unknown. Its components have to be understood in this context as algorithmic coefficients.

The norm $\left|\widehat{\mathcal{L}}\left(\boldsymbol{k}^{0}\right)\right|_{M}$ can be computed as the square root of the maximum eigenvalue (in module) of the generalized eigenvalue problem $\widehat{\mathcal{L}}\left(\boldsymbol{k}^{0}\right)^{\mathrm{t}} M \widehat{\mathcal{L}}\left(\boldsymbol{k}^{0}\right) X=\lambda M^{-1} X$. This leads to an effective way to determine the expression of matrix $\Lambda$.

The general idea exposed allows one to obtain the correct matrix of stabilization parameters for several problems (see [12] for an obtention of this matrix in the context of the hyperbolic wave equation). In particular, we will apply it now to the design of this matrix for the problem considered in this paper. Furthermore, we will show that in this particular case a simple dimensional argument is enough to obtain $\Lambda$ if we assume this matrix is diagonal.

For the sake of simplicity, let us consider the case $d=2$ (being obvious the extension to $d=3)$ and let us organize the unknowns as $U=\left(u_{1}, u_{2}, p, \sigma_{11}, \sigma_{12}, \sigma_{22}\right)$. The first point is to choose matrix $M$. If [.] denotes a dimensional group, from (3.6) it is readily checked that
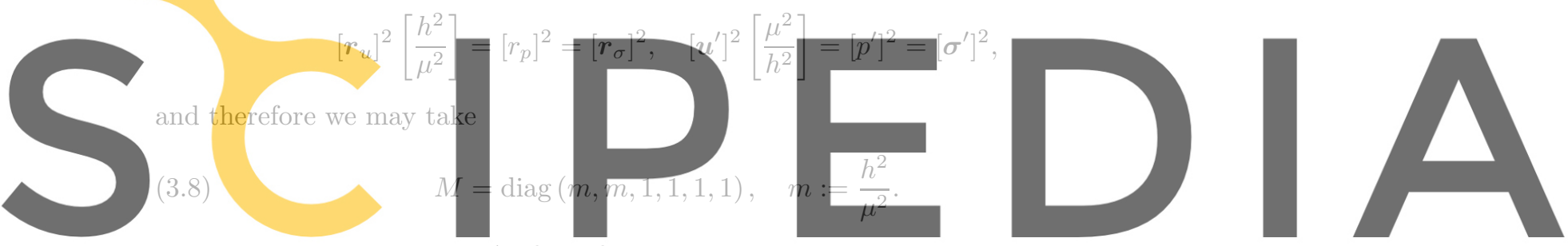

Let us consider matrix $\Lambda$ of the form

\section{Register for free at https//WWW:SCipledia, đo, m, to, download the version without the watermark}

If we apply the strategy presented above to determine $\Lambda_{u}, \Lambda_{p}$, and $\Lambda_{\sigma}$, it turns out that these parameters are uniquely determined by dimensionality. To see this, let us start by noting that if $\mathcal{L}$ is now the operator associated to (3.6), it can be checked that the eigenvalue of the problem

$$
M \widehat{\mathcal{L}}\left(\boldsymbol{k}^{0}\right)^{\mathrm{t}} M \widehat{\mathcal{L}}\left(\boldsymbol{k}^{0}\right) X=\lambda X
$$

has dimensions $[\lambda]=[\mu]^{-2}$, and therefore

$$
M \Lambda M \Lambda=\operatorname{diag}\left(\Lambda_{u}^{2} m^{2}, \Lambda_{u}^{2} m^{2}, \Lambda_{p}^{2}, \Lambda_{\sigma}^{2}, \Lambda_{\sigma}^{2}, \Lambda_{\sigma}^{2}\right)
$$

has to have all the diagonal entries of dimension $[\mu]^{-2}$. Being $\mu$ the only parameter of the equation, this immediately implies that

$$
\Lambda_{u}^{-1}=\alpha_{u} \frac{h^{2}}{\mu}, \quad \Lambda_{p}^{-1}=\alpha_{p} 2 \mu, \quad \Lambda_{\sigma}^{-1}=\alpha_{\sigma} 2 \mu,
$$

where $\alpha_{u}, \alpha_{p}$, and $\alpha_{\sigma}$ are constants that play the role of the algorithmic parameters of the formulation. This allows us to approximate the solution of (3.6) as

$$
\boldsymbol{u}_{K}^{\prime}=\alpha_{u} \frac{h^{2}}{\mu} \boldsymbol{r}_{u}
$$




$$
\begin{aligned}
p^{\prime} & =\alpha_{p} 2 \mu r_{p}, \\
\boldsymbol{\sigma}^{\prime} & =\alpha_{\sigma} 2 \mu \boldsymbol{r}_{\sigma} .
\end{aligned}
$$

These are the expressions we were looking for.

It only remains to determine which is the space of the subscales, that is, to choose the functions $\boldsymbol{\xi}_{u}, \xi_{p}$, and $\boldsymbol{\xi}_{\sigma}$. Our particular choice is to take the space for the subscales $P_{h}$ orthogonal to the finite element space (see (3.5) for the definition of $P_{h}$ ). In view of (3.9)-(3.11), this implies that $\boldsymbol{r}_{u}, r_{p}$, and $\boldsymbol{r}_{\sigma}$ must be orthogonal to $\mathcal{V}_{h}, \mathcal{Q}_{h}$, and $\mathcal{T}_{h}$, respectively. Denoting by $P_{u}, P_{p}$, and $P_{\sigma}$, the $P_{h}$ projections onto these spaces and by $P_{u}^{\perp}, P_{p}^{\perp}$, and $P_{\sigma}^{\perp}$ the orthogonal projections, we will have that

$$
\begin{aligned}
& \boldsymbol{\xi}_{u}=-P_{u}\left(\boldsymbol{f}+\nabla \cdot \boldsymbol{\sigma}_{h}-\nabla p_{h}\right) \quad \text { and } \quad \boldsymbol{u}_{K}^{\prime}=\alpha_{u} \frac{h^{2}}{\mu} P_{u}^{\perp}\left(\boldsymbol{f}+\nabla \cdot \boldsymbol{\sigma}_{h}-\nabla p_{h}\right), \\
& \xi_{p}=-P_{p}\left(-\nabla \cdot u_{h}\right) \\
& \xi_{\sigma}=-P_{\sigma}\left(-\frac{1}{2 \mu} \sigma_{h}+\nabla^{S} u_{h}\right) \quad \text { and } \quad p^{\prime}=\alpha_{p} 2 \mu P_{p}^{\perp}\left(-\nabla \cdot u_{h}\right),
\end{aligned}
$$

Clearly, we have that $P_{\sigma}^{\perp}\left(-\sigma_{h}\right)=0$. We may also assume for simplicity that the body force belongs to the finite element space, and thus $P_{u}^{\perp}(\boldsymbol{f})=0$. Hence, the expressions for the subscales we finally propose are
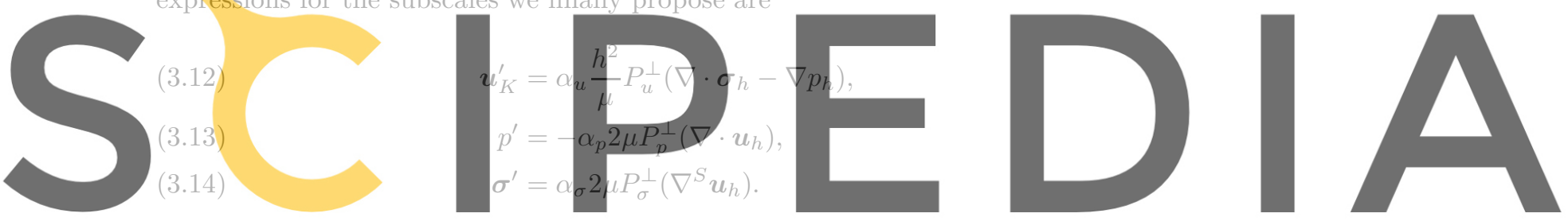

3.3. Approximation of the displacement subscale on the interelement

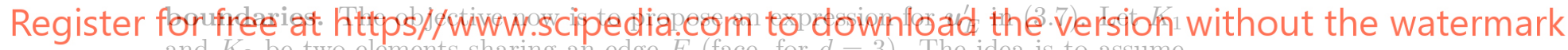
and $K_{2}$ be two elements sharing an edge $E$ (face, for $d=3$ ). The idea is to assume that the expressions (3.12)-(3.14) just obtained for $u_{K_{i}}^{\prime}, p_{i}^{\prime}$, and $\sigma_{i}^{\prime}$ on element $K_{i}$, $i=1,2$, hold up to a distance $\delta=\delta_{0} h, 0<\delta_{0}<1 / 2$, to the edge $E$, and that the normal derivative of $\boldsymbol{u}^{\prime}$ on $E$ can be approximated as

$$
\left.\boldsymbol{n}_{i} \cdot \nabla \boldsymbol{u}^{\prime}\right|_{\partial K_{i} \cap E} \approx \frac{1}{\delta}\left(\boldsymbol{u}_{E}^{\prime}-\boldsymbol{u}_{K_{i}}^{\prime}\right), \quad i=1,2,
$$

which will contribute to the stress on $\partial K_{i} \cap E$ with

$$
\left.\boldsymbol{n}_{i} \cdot \boldsymbol{\sigma}_{E}^{\prime}\right|_{\partial K_{i} \cap E}=2 \mu \boldsymbol{A}\left(\left.\boldsymbol{n}_{i} \cdot \nabla \boldsymbol{u}^{\prime}\right|_{\partial K_{i} \cap E}\right),
$$

where tangential derivatives $\boldsymbol{u}^{\prime}$ on $\partial K_{i} \cap E$ have been disregarded and $\boldsymbol{A}$ is a sym-

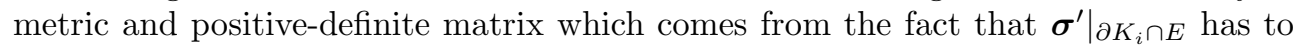
be approximated by the symmetric gradient of $\boldsymbol{u}^{\prime}$ on $\partial K_{i} \cap E$.

Calling also $\boldsymbol{u}_{K_{i}}^{\prime}, p_{i}^{\prime}$, and $\boldsymbol{\sigma}_{i}^{\prime}$, the extension of the subgrid displacement, pressure, and stress computed in the interior of element $K_{i}(i=1,2)$ and extended to the boundary, the continuity of the total stress expressed in (3.7) implies

$$
\begin{aligned}
0 & =\llbracket \boldsymbol{n}\left(p_{h}+p^{\prime}\right)-\boldsymbol{n} \cdot\left(\boldsymbol{\sigma}_{h}+\boldsymbol{\sigma}^{\prime}+\boldsymbol{\sigma}_{E}^{\prime}\right) \rrbracket_{E} \\
& =\llbracket \boldsymbol{n}\left(p_{h}+p^{\prime}\right)-\boldsymbol{n} \cdot\left(\boldsymbol{\sigma}_{h}+\boldsymbol{\sigma}^{\prime}\right) \rrbracket_{E}-2 \mu \boldsymbol{A} \llbracket \boldsymbol{n} \cdot \nabla \boldsymbol{u}^{\prime} \rrbracket_{E},
\end{aligned}
$$


and using (3.15)

$$
\boldsymbol{u}_{E}^{\prime}=\left\{\boldsymbol{u}_{K}^{\prime}\right\}_{E}+\frac{\delta}{2 \mu} \boldsymbol{A}^{-1} \llbracket \boldsymbol{n}\left(p_{h}+p^{\prime}\right)-\boldsymbol{n} \cdot\left(\boldsymbol{\sigma}_{h}+\boldsymbol{\sigma}^{\prime}\right) \rrbracket_{E},
$$

where $\left\{\boldsymbol{u}_{K}^{\prime}\right\}_{E}=\left.\left(\boldsymbol{u}_{K_{1}}^{\prime}+\boldsymbol{u}_{K_{2}}^{\prime}\right)\right|_{E} / 2$ is the average of the displacement subscales computed in the element interiors and extended to edge $E$.

Expression (3.16) can be used as subscale on the element boundaries. In fact, all the analysis presented in section 4 carries over when it is used. However, both from numerical experiments and from the numerical analysis presented later on it turns out that it suffices to use a simpler expression, obtained by keeping the dominant finite element terms in (3.16) and replacing $\boldsymbol{A}$ by the identity (recall that this is a symmetric and positive-definite matrix). The bottom line is expression

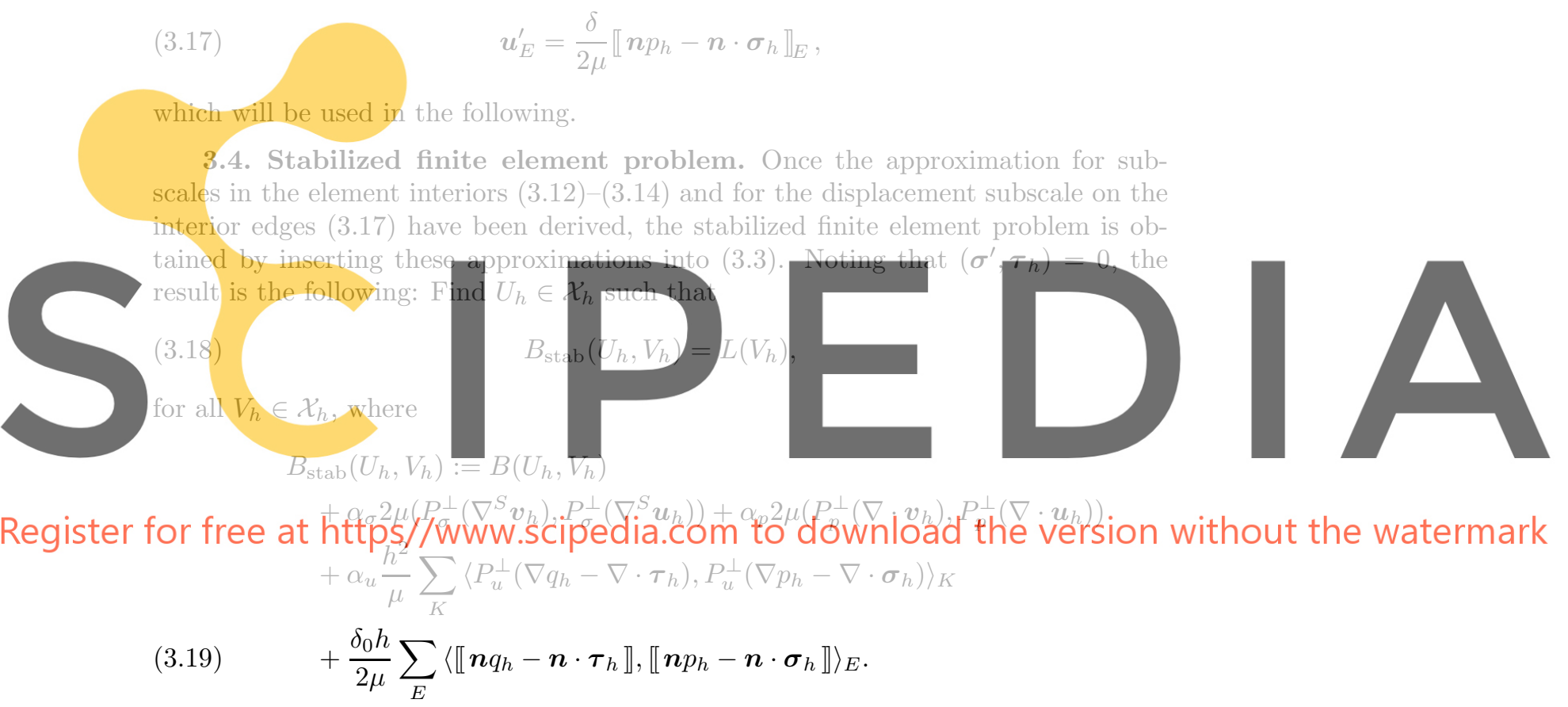

The stabilized finite element method we propose and whose stability and convergence properties are established in the following section is (3.18). In expression (3.19) for the stabilized bilinear form some orthogonal projections are used to highlight the symmetry of the resulting formulation. If $P^{\perp}$ is any of the orthogonal projections appearing in (3.19) and $P=I-P^{\perp}$, in the implementation of the method for any discrete functions $f_{h}$ and $g_{h}$ one may compute $\left(P^{\perp}\left(f_{h}\right), P^{\perp}\left(g_{h}\right)\right)=\left(f_{h}, g_{h}-P\left(g_{h}\right)\right)$ and treat $P\left(g_{h}\right)$ either implicitly or in an iterative way, that is, evaluated at a previous iteration of an iterative scheme of any type. For example, denoting with a superscript the iteration counter, in the simplest case $\left(P^{\perp}\left(f_{h}\right), P^{\perp}\left(g_{h}^{i}\right)\right)$ could be approximated by $\left(f_{h}, g_{h}^{i}-P\left(g_{h}^{i-1}\right)\right)$ (see [11] for more comments on implementation issues of a similar formulation).

Finally, let us comment on the choice of the constants $\alpha_{\sigma}, \alpha_{p}, \alpha_{u}$, and $\delta_{0}$. The analysis to be presented next can be applied for any set of values. In some numerical tests using linear and quadratic elements, with both continuous and discontinuous stresses and pressures (alhough with the same interpolation for $\boldsymbol{\sigma}_{h}$ and $p_{h}$ ) we have 
observed that these parameters can be taken in a wide range with little influence in the results. By default, we use $\alpha_{\sigma}=\alpha_{p}=1, \alpha_{u}=4$, and $\delta_{0}=1 / 10$ in our numerical tests.

4. Numerical analysis of the formulation. We present here the numerical analysis of the method proposed in the previous section using heuristic arguments. The norm in which the results will be first presented is

$$
\begin{aligned}
\left\|V_{h}\right\|^{2} & :=\frac{1}{2 \mu}\left\|\boldsymbol{\tau}_{h}\right\|^{2}+\alpha_{\sigma} 2 \mu\left\|\nabla^{S} \boldsymbol{v}_{h}\right\|^{2}+\alpha_{p} 2 \mu\left\|\nabla \cdot \boldsymbol{v}_{h}\right\|^{2} \\
& +\alpha_{u} \frac{h^{2}}{\mu} \sum_{K}\left\|\nabla q_{h}-\nabla \cdot \boldsymbol{\tau}_{h}\right\|_{K}^{2}+\delta_{0} \frac{h}{\mu} \sum_{E}\left\|\llbracket \boldsymbol{n} q_{h}-\boldsymbol{n} \cdot \boldsymbol{\tau}_{h} \rrbracket\right\|_{E}^{2},
\end{aligned}
$$

although later on we will transform our results to "natural" norms. In fact, the term multiplied by $\alpha_{p}$ is unnecessary, since it already appears in the term multiplied by $\alpha_{\sigma}$. However, we will keep it for generality, to see the effect of the subscale associated to the pressure introduced in the previous section. Moreover it would be essential in the case of some nonconforming elements (not considered in this work) for which the discrete Korn's inequality does not hold in general (see [22]). In all what follows we will assume that all the numerical parameters $\alpha_{\sigma}, \alpha_{p}, \alpha_{u}$ and $\delta_{0}$ are positive.

As it has been mentioned in section 2, we will consider for the sake of conciseness quasi-uniform finite elem

$C_{\text {inv }}$, independent of the such that

$(4.2)$

for all finite element functio
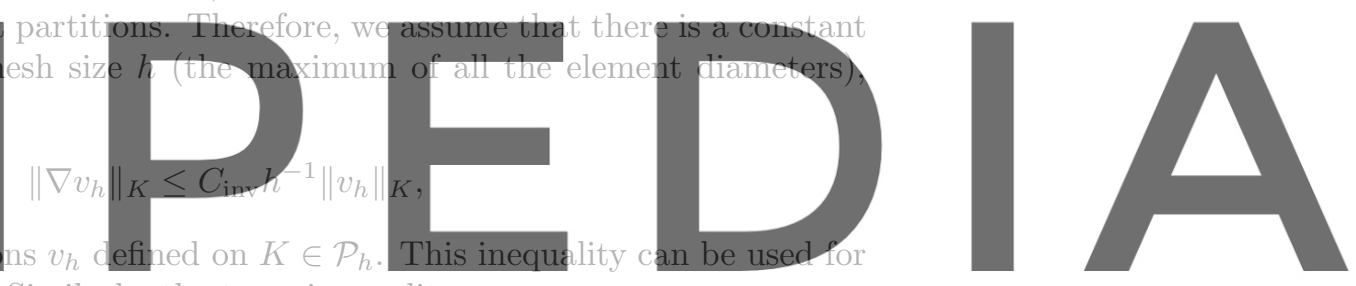

scalars, vectors, or tensors. Similarly, the trace inequality

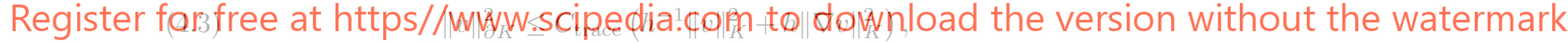

is assumed to hold for functions $v \in H^{1}(K), K \in \mathcal{P}_{h}$. The last term can be dropped if $v$ is a polynomial on the element domain $K$. Thus, if $\varphi_{h}$ is a piecewise discontinuous polynomial (the pressure or the stresses, in our case) and $\psi_{h}$ a continuous one, it follows that

$$
\begin{gathered}
\sum_{E} \|\left[\boldsymbol{n} \varphi_{h} \rrbracket \|_{E}^{2}\right. \\
\leq 2 C_{\text {trace }} h^{-1} \sum_{K}\left\|\varphi_{h}\right\|_{K}^{2}, \\
\sum_{E}\left\|\psi_{h}\right\|_{E}^{2} \leq \frac{1}{2} C_{\text {trace }} h^{-1} \sum_{K}\left\|\psi_{h}\right\|_{K}^{2} .
\end{gathered}
$$

In all what follows, $C$, with or without subscript, will denote a positive constant, independent of the discretization and the physical coefficient $\mu$, and possibly different at different occurrences.

We start proving what is in fact the key result, which states that the formulation proposed is stable in the norm (4.1). This stability is presented in the form of an inf-sup condition:

Theorem 4.1 (stability). There is a constant $C>0$ such that

$$
\inf _{U_{h} \in \mathcal{X}_{h}} \sup _{V_{h} \in \mathcal{X}_{h}} \frac{B_{\text {stab }}\left(U_{h}, V_{h}\right)}{\left\|U_{h}\right\|\left\|V_{h}\right\|} \geq C .
$$

Copyright $@$ by SIAM. Unauthorized reproduction of this article is prohibited. 
Proof. Let us start noting that, for any function $U_{h} \in \mathcal{X}_{h}$, we have

$$
\begin{gathered}
B_{\text {stab }}\left(U_{h}, U_{h}\right)=\frac{1}{2 \mu}\left\|\boldsymbol{\sigma}_{h}\right\|^{2}+\alpha_{\sigma} 2 \mu\left\|P_{\sigma}^{\perp}\left(\nabla^{S} \boldsymbol{u}_{h}\right)\right\|^{2}+\alpha_{p} 2 \mu\left\|P_{p}^{\perp}\left(\nabla \cdot \boldsymbol{u}_{h}\right)\right\|^{2} \\
+\alpha_{u} \frac{h^{2}}{\mu} \sum_{K}\left\|P_{u}^{\perp}\left(\nabla p_{h}-\nabla \cdot \boldsymbol{\sigma}_{h}\right)\right\|_{K}^{2}+\frac{\delta_{0} h}{2 \mu} \sum_{E}\left\|\llbracket \boldsymbol{n} p_{h}-\boldsymbol{n} \cdot \boldsymbol{\sigma}_{h} \rrbracket\right\|_{E}^{2} .
\end{gathered}
$$

The basic idea is to obtain control on the components on the finite element space for the terms whose orthogonal components appear in this expression. The key point is that this control comes from the Galerkin terms in the bilinear form $B_{\text {stab }}$.

Let us consider $V_{h 1}:=\alpha_{u} \frac{h^{2}}{\mu}\left(P_{u}\left(\nabla p_{h}-\nabla \cdot \boldsymbol{\sigma}_{h}\right), 0, \mathbf{0}\right)$. Recall that $P_{u}$ is defined based on elementwise integrals, and thus $P_{u}\left(\nabla p_{h}-\nabla \cdot \sigma_{h}\right)$ is well defined. We will use the abbreviation $\boldsymbol{v}_{1} \equiv P_{u}\left(\nabla p_{h}-\nabla \cdot \boldsymbol{\sigma}_{h}\right)$. A straightforward application of Schwarz's inequality and the inverse estimate (4.2) leads to

$$
\begin{aligned}
B_{\text {stab }}\left(U_{h}, V_{h 1}\right) & \geq B\left(U_{h}, V_{h 1}\right)-\alpha_{\sigma} 2 \mu \alpha_{u} \frac{h^{2}}{\mu} \frac{C_{\text {inv }}}{h}\left\|\boldsymbol{v}_{1}\right\|\left\|P_{\sigma}^{\perp}\left(\nabla^{S} \boldsymbol{u}_{h}\right)\right\| \\
& -\alpha_{p} 2 \mu \alpha_{u} \frac{h^{2}}{\mu} \frac{C_{\text {inv }}}{h}\left\|\boldsymbol{v}_{1}\right\|\left\|P_{p}^{\perp}\left(\nabla \cdot \boldsymbol{u}_{h}\right)\right\| .
\end{aligned}
$$

On the other hand,

$$
\begin{aligned}
& B\left(U_{h}, V_{h 1}\right)=\alpha_{u} \frac{h^{2}}{\mu} \sum_{K}\left(\left\langle\nabla^{S} \boldsymbol{v}_{1}, \boldsymbol{\sigma}_{h}\right\rangle_{K}-\left\langle\nabla \cdot \boldsymbol{v}_{1}, p_{h}\right\rangle_{K}\right) \\
& \quad=\alpha_{u} \frac{h^{2}}{\mu} \sum_{K}\left(-\left\langle\boldsymbol{v}_{1}, \nabla \cdot \boldsymbol{\sigma}_{h}\right\rangle_{K}+\left\langle\boldsymbol{v}_{1}, \nabla p_{h}\right\rangle_{K}\right)-\alpha_{u} \frac{h^{2}}{\mu} \sum_{E}\left\langle\boldsymbol{v}_{1}, \llbracket \boldsymbol{n} p_{h}-\boldsymbol{n} \cdot \boldsymbol{\sigma}_{h} \rrbracket\right\rangle_{E} \\
& \quad \geq \alpha_{u} \frac{h^{2}}{\mu} \sum_{K}\left\|\boldsymbol{v}_{1}\right\|_{K}^{2}-\alpha_{u} \frac{h^{2}}{\mu} \sum_{E}\left\|\boldsymbol{v}_{1}\right\|_{E}\left\|\llbracket \boldsymbol{n} p_{h}-\boldsymbol{n} \cdot \boldsymbol{\sigma}_{h} \rrbracket\right\|_{E} \\
& \geq \alpha_{u} \frac{h^{2}}{2 \mu} \sum_{K}\left\|\boldsymbol{v}_{1}\right\|_{K}^{2}-\alpha_{u} \frac{h C_{\text {trace }}}{4 \mu} \sum_{E}\left\|\llbracket \boldsymbol{n} p_{h}-\boldsymbol{n} \cdot \boldsymbol{\sigma}_{h} \rrbracket\right\|_{E}^{2},
\end{aligned}
$$

where Young's inequality and (4.5) have been used in the last step. Using this in (4.8) and making use again of Young's inequality, it follows that there exist constants $C_{1 j}$, $j=1,2,3,4$, such that

$$
\begin{aligned}
B_{\text {stab }}\left(U_{h}, V_{h 1}\right) & \geq C_{11} \alpha_{u} \frac{h^{2}}{\mu}\left\|P_{u}\left(\nabla p_{h}-\nabla \cdot \boldsymbol{\sigma}_{h}\right)\right\|^{2}-C_{12} \alpha_{u} \frac{h}{\mu} \sum_{E}\left\|\llbracket \boldsymbol{n} p_{h}-\boldsymbol{n} \cdot \boldsymbol{\sigma}_{h} \rrbracket\right\|_{E}^{2} \\
& -C_{13} \alpha_{u} \alpha_{\sigma}^{2} \mu\left\|P_{\sigma}^{\perp}\left(\nabla^{S} \boldsymbol{u}_{h}\right)\right\|^{2}-C_{14} \alpha_{u} \alpha_{p}^{2} \mu\left\|P_{p}^{\perp}\left(\nabla \cdot \boldsymbol{u}_{h}\right)\right\|^{2} .
\end{aligned}
$$

Consider now $V_{h 2}:=\alpha_{p} 2 \mu\left(\mathbf{0}, q_{2}, \mathbf{0}\right)$, where $q_{2} \equiv P_{p}\left(\nabla \cdot \boldsymbol{u}_{h}\right)$. Note that this function may be discontinuous across interelement boundaries. It turns out that

$$
\begin{aligned}
B_{\text {stab }}\left(U_{h}, V_{h 2}\right) & =\alpha_{p} 2 \mu\left\|q_{2}\right\|^{2}+\alpha_{u} \frac{h^{2}}{2 \mu} \sum_{K}\left\langle\nabla q_{2}, P_{u}^{\perp}\left(\nabla p_{h}-\nabla \cdot \boldsymbol{\sigma}_{h}\right)\right\rangle_{K} \\
& +\delta_{0} \frac{h}{\mu} \alpha_{p} 2 \mu \sum_{E}\left\langle\llbracket q_{2} \rrbracket, \llbracket \boldsymbol{n} p_{h}-\boldsymbol{n} \cdot \boldsymbol{\sigma}_{h} \rrbracket\right\rangle_{E}
\end{aligned}
$$

Copyright $\odot$ by SIAM. Unauthorized reproduction of this article is prohibited. 
The same strategy as before, now using (4.4) to deal with the last term in this expression, leads to the existence of certain constants $C_{2 j}, j=1,2,3$, such that

$$
\begin{aligned}
B_{\mathrm{stab}}\left(U_{h}, V_{h 2}\right) & \geq C_{21} \alpha_{p} \mu\left\|P_{p}\left(\nabla \cdot \boldsymbol{u}_{h}\right)\right\|^{2}-C_{22} \alpha_{p} \alpha_{u}^{2} \frac{h^{2}}{\mu}\left\|P_{u}^{\perp}\left(\nabla p_{h}-\nabla \cdot \boldsymbol{\sigma}_{h}\right)\right\|^{2} \\
& -C_{23} \alpha_{p} \delta_{0}^{2} \frac{h}{\mu} \sum_{E}\left\|\llbracket \boldsymbol{n} p_{h}-\boldsymbol{n} \cdot \boldsymbol{\sigma}_{h} \rrbracket\right\|_{E}^{2} .
\end{aligned}
$$

Finally, taking $V_{h 3}:=\alpha_{\sigma} 2 \mu\left(\mathbf{0}, 0,-P_{\sigma}\left(\nabla^{S} \boldsymbol{u}_{h}\right)\right)$ we obtain that there exist constants $C_{3 j}, j=1,2,3,4$, such that

$$
\begin{aligned}
& B_{\text {stab }}\left(U_{h}, V_{h 3}\right) \geq C_{31} \alpha_{\sigma} \mu\left\|P_{\sigma}\left(\nabla^{S} \boldsymbol{u}_{h}\right)\right\|^{2}-C_{32} \alpha_{\sigma} \frac{1}{\mu}\left\|\boldsymbol{\sigma}_{h}\right\|^{2} \\
& \left.\quad-C_{33} \alpha_{\sigma} \alpha_{u}^{2} \frac{h^{2}}{\mu}\left\|P_{u}^{\perp}\left(\nabla p_{h}-\nabla \cdot \boldsymbol{\sigma}_{h}\right)\right\|^{2}-C_{34} \alpha_{\sigma} \delta_{0}^{2} \frac{h}{\mu} \sum_{E} \| \llbracket \boldsymbol{n} p_{h}-\boldsymbol{n} \cdot \boldsymbol{\sigma}_{h}\right] \|_{E}^{2} .
\end{aligned}
$$

Let $V_{h}=U_{h}+\beta_{1} V_{h 1}+\beta_{2} V_{h 2}+\beta_{3} V_{h 3}$, with $V_{h i}, i=1,2,3$, introduced above. Adding up inequalities (4.9)-(4.11) multiplied by $\beta_{1}, \beta_{2}$, and $\beta_{3}$, respectively, and adding also (4.7), it is trivially verified that the coefficients $\beta_{i}, i=1,2,3$, can be chosen large enough so as to obtain

$$
B_{\text {stab }}\left(U_{h}, V_{h}\right) \geq C\left\|U_{h}\right\|^{2}
$$

On the other hand, we have that

$$
\begin{aligned}
\left\|V_{h 1}\right\|^{2} & \leq 2 \alpha_{u}^{2}\left(\alpha_{p}+\alpha_{\sigma}\right) C_{\mathrm{inv}}^{2} \frac{h^{2}}{\mu}\left\|\nabla p_{h}-\nabla \cdot \boldsymbol{\sigma}_{h}\right\|^{2} \leq C\left\|U_{h}\right\|^{2}, \\
\left\|V_{h 2}\right\|^{2} & \leq 2 \mu \alpha_{p}^{2}\left(2 \alpha_{u} C_{\mathrm{inv}}^{2}+4 \delta_{0} C_{\text {trace }}\right)\left\|\nabla \cdot \boldsymbol{u}_{h}\right\|^{2} \leq C\left\|U_{h}\right\|^{2} \\
\left\|V_{h 3}\right\|^{2} & \leq 2 \alpha_{\sigma}^{2} \mu\left(1+2 \alpha_{u} C_{\mathrm{inv}}^{2}+4 \delta_{0} C_{\text {trace }}\right)\left\|\nabla^{S} \boldsymbol{u}_{h}\right\|^{2} \leq C\left\|U_{h}\right\|^{2},
\end{aligned}
$$

from where it follows that $\left\|V_{h}\right\| \leq C\left\|U_{h}\right\|$. Using this fact in (4.12) we have shown that for each $U_{h} \in \mathcal{X}_{h}$ there exists $V_{h} \in \mathcal{X}_{h}$ such that $B_{\text {stab }}\left(U_{h}, V_{h}\right) \geq C\left\|U_{h}\right\|\left\|V_{h}\right\|$, from where the theorem follows.

Once stability is established, a more or less standard procedure leads to convergence. To prove it, we need two preliminary lemmas. The first concerns the consistency of the formulation:

LEMMA 4.2 (consistency). Let $U \in \mathcal{X}$ be the solution of the continuous problem and $U_{h} \in \mathcal{X}_{h}$ the finite element solution of (3.18). If $\boldsymbol{f} \in \mathcal{V}_{h}$ and $U$ is regular enough, so that $B_{\text {stab }}\left(U, V_{h}\right)$ is well defined, then

$$
B_{\text {stab }}\left(U-U_{h}, V_{h}\right)=0 \quad \forall V_{h} \in \mathcal{X}_{h}
$$

Proof. This lemma is a trivial consequence of the consistency of the finite element method proposed (considering the force term $\boldsymbol{f}$ in the finite element space). Note

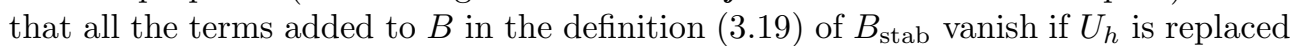
by $U$ (recall that $\boldsymbol{\sigma}_{h}$ could have been added to $\nabla^{S} \boldsymbol{u}_{h}$, since $P_{\sigma}^{\perp}\left(\boldsymbol{\sigma}_{h}\right)=\mathbf{0}$ ).

Remark 4.1. If $P_{u}^{\perp}(\boldsymbol{f}) \neq \mathbf{0}$ there are two options. The first is to include this orthogonal projection in the definition of the method, and therefore to modify the right-hand side of (3.18). All the analysis carries over to this case. The second is to take into account the consistency error coming from $\boldsymbol{f}$ in (4.13). It is easy to see that 
in this case this equation can be replaced by $B_{\mathrm{stab}}\left(U-U_{h}, V_{h}\right) \leq C E(h)\left\|V_{h}\right\|$, where $E(h)$ is introduced below, and the following results can be immediately adapted.

The second preliminary lemma concerns an interpolation error in terms of the norm $\|\cdot\|$ and the bilinear form $B_{\text {stab }}$ for the continuous solution $U=(\boldsymbol{u}, p, \boldsymbol{\sigma}) \in \mathcal{X}$, assumed to have enough regularity. Let $\mathcal{W}_{h}$ be a finite element space of degree $k_{v}$. For any function $v \in H^{k_{v}^{\prime}+1}(\Omega)$ and for $i=0,1$, we define the interpolation errors $\varepsilon_{i}(v)$ from the interpolation estimates

$$
\inf _{v_{h} \in \mathcal{W}_{h}} \sum_{K}\left\|v-v_{h}\right\|_{H^{i}(K)} \leq C h^{k_{v}^{\prime \prime}+1-i} \sum_{K}\|v\|_{H^{k_{v}^{\prime \prime}+1}(K)}=: \varepsilon_{i}(v),
$$

where $k_{v}^{\prime \prime}=\min \left(k_{v}, k_{v}^{\prime}\right)$. We will denote by $\tilde{v}_{h}$ the best approximation of $v$ in $\mathcal{W}_{h}$. Clearly, we have that $\varepsilon_{0}(v)=h \varepsilon_{1}(v)$. We will use this notation for $v=\boldsymbol{u}$ (displacement), $v=p$ (pressure) and $v=\boldsymbol{\sigma}$ (stresses), being the respective orders of interpolation $k_{u}, k_{p}$ and $k_{\sigma}$.

This notation will allow us to prove that the error function of the method is

$$
E(h):=\sqrt{\mu} \varepsilon_{1}(\boldsymbol{u})+\frac{1}{\sqrt{\mu}} \varepsilon_{0}(p)+\frac{1}{\sqrt{\mu}} \varepsilon_{0}(\boldsymbol{\sigma}) .
$$

This is indeed the interpolation error:

Lemma 4.3 (interpolation error). Let $U \in \mathcal{X}$ be the continuous solution, assumed to be regular enough, and $\tilde{U}_{h} \in \mathcal{X}_{h}$ its best finite element approximation. Then, the following inequalities hold:

$$
\begin{aligned}
& B_{\text {stab }}\left(U-\tilde{U}_{h}, V_{h}\right) \leq C E(h)\left\|V_{h}\right\|, \\
& \left\|U-\tilde{U}_{h}\right\| \leq C E(h),
\end{aligned}
$$

where $E(h)$ is given in (4.15).

Proof. Let us start considering a general discontinuous finite element interpolation of a function $v$. Using the trace inequality (4.3) we have that

$$
\begin{aligned}
\left.\sum_{E} \| \llbracket \boldsymbol{n}\left(v-\tilde{v}_{h}\right)\right] \|_{E}^{2} & \leq 2 \sum_{K}\left\|v-\tilde{v}_{h}\right\|_{\partial K}^{2} \\
& \leq 2 C_{\text {trace }} \sum_{K}\left(h^{-1}\left\|v-\tilde{v}_{h}\right\|_{K}^{2}+h\left\|\nabla v-\nabla \tilde{v}_{h}\right\|_{K}^{2}\right) \\
& \leq C\left(h^{-1} \varepsilon_{0}^{2}(v)+h \varepsilon_{1}^{2}(v)\right) .
\end{aligned}
$$

The same estimate holds for a continuous interpolation:

$$
\sum_{E}\left\|\left(v-\tilde{v}_{h}\right)\right\|_{E}^{2} \leq C\left(h^{-1} \varepsilon_{0}^{2}(v)+h \varepsilon_{1}^{2}(v)\right) .
$$

Let us prove (4.17). By the definition (4.1) of the norm $\|\cdot\|$ and the result just obtained it is immediately checked that

$$
\begin{aligned}
\left\|U-\tilde{U}_{h}\right\|^{2} & \leq C\left[\frac{1}{2 \mu} \varepsilon_{0}^{2}(\boldsymbol{\sigma})+\alpha_{\sigma} 2 \mu \varepsilon_{1}^{2}(\boldsymbol{u})+\alpha_{p} 2 \mu \varepsilon_{1}^{2}(\boldsymbol{u})\right. \\
& \left.+\alpha_{u} \frac{h^{2}}{\mu} \varepsilon_{1}^{2}(p)+\alpha_{u} \frac{h^{2}}{\mu} \varepsilon_{1}^{2}(\boldsymbol{\sigma})+\delta_{0} \frac{h^{2}}{\mu} \varepsilon_{1}^{2}(p)+\delta_{0} \frac{h^{2}}{\mu} \varepsilon_{1}^{2}(\boldsymbol{\sigma})\right],
\end{aligned}
$$

and (4.17) follows. 
Let $\boldsymbol{e}_{u}=\boldsymbol{u}-\tilde{\boldsymbol{u}}_{h}, e_{p}=p-\tilde{p}_{h}$, and $\boldsymbol{e}_{\sigma}=\boldsymbol{\sigma}-\tilde{\boldsymbol{\sigma}}_{h}$. The proof of (4.16) is as follows:

$$
\begin{aligned}
B_{\text {stab }} & \left(U-\tilde{U}_{h}, V_{h}\right)=\left(\nabla^{S} \boldsymbol{v}_{h}, \boldsymbol{e}_{\sigma}\right)-\left(e_{p}, \nabla \cdot \boldsymbol{v}_{h}\right)+\frac{1}{2 \mu}\left(\boldsymbol{\tau}_{h}, \boldsymbol{e}_{\sigma}\right) \\
& -\sum_{K}\left\langle-\nabla q_{h}+\nabla \cdot \boldsymbol{\tau}_{h}, \boldsymbol{e}_{u}\right\rangle_{K}+\sum_{E}\left\langle\llbracket \boldsymbol{n} q_{h}-\boldsymbol{n} \cdot \boldsymbol{\tau}_{h} \rrbracket, \boldsymbol{e}_{u}\right\rangle_{E} \\
& +\alpha_{\sigma}\left(P_{\sigma}^{\perp}\left(\nabla^{S} \boldsymbol{v}_{h}\right), P_{\sigma}^{\perp}\left(2 \mu \nabla^{S} \boldsymbol{e}_{u}-\boldsymbol{e}_{\sigma}\right)\right)+\alpha_{p} 2 \mu\left(P_{\sigma}^{\perp}\left(\nabla \cdot \boldsymbol{v}_{h}\right), P_{\sigma}^{\perp}\left(\nabla \cdot \boldsymbol{e}_{u}\right)\right) \\
& +\delta_{0} \frac{h}{\mu} \sum_{E}\left\langle\llbracket \boldsymbol{n} q_{h}-\boldsymbol{n} \cdot \boldsymbol{\tau}_{h} \rrbracket, \llbracket \boldsymbol{n} e_{p}-\boldsymbol{n} \cdot \boldsymbol{e}_{\sigma} \rrbracket\right\rangle_{E} \\
\leq & C\left[\sqrt{\mu}\left\|\nabla^{S} \boldsymbol{v}_{h}\right\| \frac{1}{\sqrt{\mu}}\left\|\boldsymbol{e}_{\sigma}\right\|+\sqrt{\mu}\left\|\nabla \cdot \boldsymbol{v}_{h}\right\| \frac{1}{\sqrt{\mu}}\left\|e_{p}\right\|+\frac{1}{2 \sqrt{\mu}}\left\|\boldsymbol{\tau}_{h}\right\| \frac{1}{\sqrt{\mu}}\left\|\boldsymbol{e}_{\sigma}\right\|\right. \\
& +\sum_{K} \frac{h}{\sqrt{\mu}}\left\|\nabla q_{h}-\nabla \cdot \boldsymbol{\tau}_{h}\right\|_{K} \frac{\sqrt{\mu}}{h}\left\|\boldsymbol{e}_{u}\right\|_{K}+\sum_{E} \frac{\sqrt{h}}{\sqrt{\mu}}\left\|\llbracket \boldsymbol{n} q_{h}-\boldsymbol{n} \cdot \boldsymbol{\tau}_{h} \rrbracket\right\|_{E} \frac{\sqrt{\mu}}{\sqrt{h}}\left\|\boldsymbol{e}_{u}\right\|_{E} \\
& +\sqrt{\mu}\left\|\nabla^{S} \boldsymbol{v}_{h}\right\| \sqrt{\mu}\left\|\nabla^{S} \boldsymbol{e}_{u}\right\|+\sqrt{\mu}\left\|\nabla^{S} \boldsymbol{v}_{h}\right\| \frac{1}{\sqrt{\mu}}\left\|\boldsymbol{e}_{\sigma}\right\|+\sqrt{\mu}\left\|\nabla \cdot \boldsymbol{v}_{h}\right\| \sqrt{\mu}\left\|\nabla \cdot \boldsymbol{e}_{u}\right\| \\
& \left.+\sum_{E} \frac{\sqrt{h}}{\sqrt{\mu}}\left\|\llbracket \boldsymbol{n} q_{h}-\boldsymbol{n} \cdot \boldsymbol{\tau}_{h} \rrbracket\right\| \|_{E} \frac{\sqrt{h}}{\sqrt{\mu}}\left(\left\|\llbracket \boldsymbol{n} e_{p} \rrbracket\right\|\left\|_{E}+\right\| \llbracket \boldsymbol{n} \cdot \boldsymbol{e}_{\sigma} \rrbracket\|\|_{E}\right)\right] .
\end{aligned}
$$

All the terms have been organized to see that, after making use of (4.18) and (4.19), they are all bounded by $C E(h)\left\|V_{h}\right\|$, from where (4.16) follows.

We are finally in a position to prove convergence. The proof is standard, but we include it for completeness.

TheOrem 4.4 (convergence). Let $U=(\boldsymbol{u}, p, \boldsymbol{\sigma}) \in \mathcal{X}$ be the solution of the continuous problem. Then, there is a constant $C>0$ such that

$$
\left\|U-U_{h}\right\| \leq C E(h),
$$

where $E(h)$ is given in (4.15).

Proof. Consider the finite element function $\tilde{U}_{h}-U_{h} \in \mathcal{X}_{h}$ where, as in Lemma 4.3, $\tilde{U}_{h} \in \mathcal{X}_{h}$ is the best finite element approximation to $U$. Starting from the inf-sup condition (4.6), it follows that there exists $V_{h} \in \mathcal{X}_{h}$ such that

$$
\begin{aligned}
C\left\|\tilde{U}_{h}-U_{h}\right\|\left\|V_{h}\right\| & \leq B_{\text {stab }}\left(\tilde{U}_{h}-U_{h}, V_{h}\right) & & \\
& =B_{\text {stab }}\left(\tilde{U}_{h}-U, V_{h}\right) & & (\text { from the consistency }(4.13)) \\
& \leq C E(h)\left\|V_{h}\right\| & & (\text { from }(4.16))
\end{aligned}
$$

from where $\left\|\tilde{U}_{h}-U_{h}\right\| \leq C E(h)$. The theorem follows now from the triangle inequality $\left\|U-U_{h}\right\| \leq\left\|U-\tilde{U}_{h}\right\|+\left\|\tilde{U}_{h}-U_{h}\right\|$ and the interpolation error estimate (4.17).

Clearly, this convergence result is optimal.

Remark 4.2. From the expression of the error function (4.15) it follows that all the terms have the same order in $h$ if $k_{u}=k_{p}+1=k_{\sigma}+1$. However, Theorem 4.4 holds without any restriction on the interpolation order of the different unknowns.

The next step will be to prove stability and convergence in natural norms, that is to say, in the norm of the space where the continuous problem is posed, and not in the mesh dependent norm (4.1). Even though the results to be presented are the 
expected ones, the analysis presented up to this point has highlighted the role played by the stabilization terms of the formulation.

THEOREM 4.5 (stability and convergence in natural norms). The solution of the discrete problem $U_{h}=\left(\boldsymbol{u}_{h}, p_{h}, \boldsymbol{\sigma}_{h}\right) \in \mathcal{X}_{h}$ can be bounded as

$$
\sqrt{\mu}\left\|\boldsymbol{u}_{h}\right\|_{H^{1}(\Omega)}+\frac{1}{\sqrt{\mu}}\left\|\boldsymbol{\sigma}_{h}\right\|+\frac{1}{\sqrt{\mu}}\left\|p_{h}\right\| \leq \frac{C}{\sqrt{\mu}}\|\boldsymbol{f}\|_{H^{-1}(\Omega)} .
$$

Moreover, if the solution of the continuous problem $U=(\boldsymbol{u}, p, \boldsymbol{\sigma}) \in \mathcal{X}$ is regular enough, the following error estimate holds:

$$
\sqrt{\mu}\left\|\boldsymbol{u}-\boldsymbol{u}_{h}\right\|_{H^{1}(\Omega)}+\frac{1}{\sqrt{\mu}}\left\|\boldsymbol{\sigma}-\boldsymbol{\sigma}_{h}\right\|+\frac{1}{\sqrt{\mu}}\left\|p-p_{h}\right\| \leq C E(h) .
$$

Proof. Let us first recall that Korn's inequality implies that $\left\|\nabla^{S} \boldsymbol{v}\right\|$ is a norm in $\mathcal{V}$ equivalent to $\|\boldsymbol{v}\|_{H^{1}(\Omega)}$, and this property is inherited by the conforming approximation considered. On the other hand, it is clear that

$$
\left\langle\boldsymbol{f}, \boldsymbol{v}_{h}\right\rangle \leq \frac{C}{\sqrt{\mu}}\|\boldsymbol{f}\|_{H^{-1}(\Omega)} \sqrt{\mu}\left\|\boldsymbol{v}_{h}\right\|_{H^{1}(\Omega)} \leq \frac{C}{\sqrt{\mu}}\|\boldsymbol{f}\|_{H^{-1}(\Omega)}\left\|V_{h}\right\|,
$$

where $V_{h}=\left(\boldsymbol{v}_{h}, q_{h}, \boldsymbol{\tau}_{h}\right) \in \mathcal{X}_{h}$ is arbitrary. Therefore the inf-sup condition proved in Theorem 4.1 implies that $\left\|U_{h}\right\| \leq \frac{C}{\sqrt{\mu}}\|\boldsymbol{f}\|_{H^{-1}(\Omega)}$, which, together with the definition of $\|\cdot\| \|$ in (4.1), yields the bound (4.20) for the first two terms in the left-hand side of this inequality. More precisely, we have that

$$
\begin{aligned}
\mu\left\|\boldsymbol{u}_{h}\right\|_{H^{1}(\Omega)}^{2}+\frac{1}{\mu}\left\|\boldsymbol{\sigma}_{h}\right\|^{2} & \\
+ & \frac{h^{2}}{\mu} \sum_{K}\left\|\nabla p_{h}-\nabla \cdot \boldsymbol{\sigma}_{h}\right\|_{K}^{2}+\frac{h}{\mu} \sum_{E}\left\|\llbracket \boldsymbol{n} p_{h}-\boldsymbol{n} \cdot \boldsymbol{\sigma}_{h} \rrbracket\right\|_{E}^{2} \leq \frac{C}{\mu}\|\boldsymbol{f}\|_{H^{-1}(\Omega)}^{2} .
\end{aligned}
$$

On the other hand, using the inverse estimate (4.2) and the trace inequality (4.3) we have

$$
\begin{aligned}
& \frac{h^{2}}{\mu} \sum_{K}\left\|\nabla p_{h}\right\|_{K}^{2} \leq \frac{h^{2}}{\mu} \sum_{K}\left\|\nabla p_{h}-\nabla \cdot \boldsymbol{\sigma}_{h}\right\|_{K}^{2}+\frac{C}{\mu}\left\|\boldsymbol{\sigma}_{h}\right\|^{2}, \\
& \frac{h}{\mu} \sum_{E}\left\|\llbracket \boldsymbol{n} p_{h} \rrbracket\right\|_{E}^{2} \leq \frac{h}{\mu} \sum_{E}\left\|\llbracket \boldsymbol{n} p_{h}-\boldsymbol{n} \cdot \boldsymbol{\sigma}_{h} \rrbracket\right\|_{E}^{2}+\frac{C}{\mu}\left\|\boldsymbol{\sigma}_{h}\right\|^{2},
\end{aligned}
$$

so that (4.22) implies

$$
\mu\left\|\boldsymbol{u}_{h}\right\|_{H^{1}(\Omega)}^{2}+\frac{1}{\mu}\left\|\boldsymbol{\sigma}_{h}\right\|^{2}+\frac{h^{2}}{\mu} \sum_{K}\left\|\nabla p_{h}\right\|_{K}^{2}+\frac{h}{\mu} \sum_{E}\left\|\llbracket \boldsymbol{n} p_{h} \rrbracket\right\|_{E}^{2} \leq \frac{C}{\mu}\|\boldsymbol{f}\|_{H^{-1}(\Omega)}^{2} .
$$

To prove the $L^{2}$-stability for the pressure we rely on the inf-sup condition between the velocity and pressure spaces that holds for the continuous problem, that is to say, the continuous counterpart of (2.9). If $p_{h}$ is the solution of the discrete problem, there exists $\boldsymbol{w} \in \mathcal{V}$ such that

$$
C\left\|p_{h}\right\|\|\boldsymbol{w}\|_{H^{1}(\Omega)} \leq\left(p_{h}, \nabla \cdot \boldsymbol{w}\right) .
$$

Copyright $@$ by SIAM. Unauthorized reproduction of this article is prohibited. 
Let us choose $\boldsymbol{w}$ with $\|\boldsymbol{w}\|_{H^{1}(\Omega)}=\left\|p_{h}\right\|$ and let $\tilde{\boldsymbol{w}}_{h}$ be the best approximation to $\boldsymbol{w}$ in $\mathcal{V}_{h}$, which will satisfy $\left\|\boldsymbol{w}-\tilde{\boldsymbol{w}}_{h}\right\| \leq C h\left\|p_{h}\right\|$. Using (4.3) once again we have that

$$
\begin{aligned}
C\left\|p_{h}\right\|^{2} \leq & \left(p_{h}, \nabla \cdot \boldsymbol{w}\right) \\
= & -\sum_{K}\left\langle\nabla p_{h}, \boldsymbol{w}-\tilde{\boldsymbol{w}}_{h}\right\rangle_{K}+\sum_{E}\left\langle\llbracket \boldsymbol{n} p_{h} \rrbracket, \boldsymbol{w}-\tilde{\boldsymbol{w}}_{h}\right\rangle_{E} \\
& +\left(\boldsymbol{\sigma}_{h}, \nabla^{S} \tilde{\boldsymbol{w}}_{h}\right)-\left\langle\boldsymbol{f}, \tilde{\boldsymbol{w}}_{h}\right\rangle \\
\leq & C\left\|p_{h}\right\|\left(h \sum_{K}\left\|\nabla p_{h}\right\|_{K}+\sqrt{h} \sum_{E}\left\|\llbracket \boldsymbol{n} p_{h} \rrbracket\right\|_{E}+\left\|\boldsymbol{\sigma}_{h}\right\|+\|\boldsymbol{f}\|_{H^{-1}(\Omega)}\right) .
\end{aligned}
$$

This, together with (4.23), implies the stability estimate (4.20).

The error estimate can be proved using a similar strategy. First, let us notice that Theorem 4.4 implies the error estimate (4.21) for the displacement and the stresses. We thus have

$$
\begin{aligned}
\mu \| \boldsymbol{u} & -\boldsymbol{u}_{h}\left\|_{H^{1}(\Omega)}^{2}+\frac{1}{\mu}\right\| \boldsymbol{\sigma}-\boldsymbol{\sigma}_{h} \|^{2} \\
& +\frac{h^{2}}{\mu} \sum_{K}\left\|\nabla\left(p-p_{h}\right)-\nabla \cdot\left(\boldsymbol{\sigma}-\boldsymbol{\sigma}_{h}\right)\right\|_{K}^{2} \\
& +\frac{h}{\mu} \sum_{E}\left\|\llbracket \boldsymbol{n}\left(p-p_{h}\right)-\boldsymbol{n} \cdot\left(\boldsymbol{\sigma}-\boldsymbol{\sigma}_{h}\right) \rrbracket\right\|_{E}^{2} \leq C E(h)^{2} .
\end{aligned}
$$

On the other hand, using the interpolation estimates (4.14) and (4.18)

$$
\begin{aligned}
& \frac{h^{2}}{\mu} \sum_{K}\left\|\nabla\left(p-p_{h}\right)\right\|_{K}^{2} \leq \frac{h^{2}}{\mu} \sum_{K}\left\|\nabla\left(p-p_{h}\right)-\nabla \cdot\left(\boldsymbol{\sigma}-\boldsymbol{\sigma}_{h}\right)\right\|_{K}^{2}+\frac{C}{\mu} \varepsilon_{0}^{2}(\boldsymbol{\sigma}), \\
& \frac{h}{\mu} \sum_{E}\left\|\llbracket \boldsymbol{n}\left(p-p_{h}\right) \rrbracket\right\|_{E}^{2} \leq \frac{h}{\mu} \sum_{E}\left\|\llbracket \boldsymbol{n}\left(p-p_{h}\right)-\boldsymbol{n} \cdot\left(\boldsymbol{\sigma}-\boldsymbol{\sigma}_{h}\right) \rrbracket\right\|_{E}^{2}+\frac{C}{\mu} \varepsilon_{0}^{2}(\boldsymbol{\sigma}),
\end{aligned}
$$

and, according to (4.24), both terms are bounded by $E(h)^{2}$. To prove the $L^{2}$-error estimate for the pressure, let now $\boldsymbol{w} \in \mathcal{V}$, with $\|\boldsymbol{w}\|_{H^{1}(\Omega)}=\left\|p-p_{h}\right\|$, be such that $C\left\|p-p_{h}\right\|^{2} \leq\left(p-p_{h}, \nabla \cdot \boldsymbol{w}\right)$, and let $\tilde{\boldsymbol{w}}_{h}$ be its best approximation in $\mathcal{V}_{h}$. We have that

$$
\begin{aligned}
C \| p & -p_{h} \|^{2} \leq\left(p-p_{h}, \nabla \cdot \boldsymbol{w}\right) \\
= & -\sum_{K}\left\langle\nabla\left(p-p_{h}\right), \boldsymbol{w}-\tilde{\boldsymbol{w}}_{h}\right\rangle_{K}+\sum_{E}\left\langle\llbracket \boldsymbol{n}\left(p-p_{h}\right) \rrbracket, \boldsymbol{w}-\tilde{\boldsymbol{w}}_{h}\right\rangle_{E} \\
& +\left(\boldsymbol{\sigma}-\boldsymbol{\sigma}_{h}, \nabla^{S} \tilde{\boldsymbol{w}}_{h}\right) \\
\leq & C\left\|p-p_{h}\right\|\left(h \sum_{K}\left\|\nabla\left(p-p_{h}\right)\right\|_{K}+\sqrt{h} \sum_{E}\left\|\llbracket \boldsymbol{n}\left(p-p_{h}\right) \rrbracket\right\|_{E}+\left\|\boldsymbol{\sigma}-\boldsymbol{\sigma}_{h}\right\|\right),
\end{aligned}
$$

which yields $\left\|p-p_{h}\right\| \leq C \sqrt{\mu} E(h)$. This, together with (4.24), finishes the proof of (4.21).

To complete the analysis of the problem, let us obtain an $L^{2}$-error estimate for the displacement, which can be proved using a duality argument.

TheOREM 4.6 ( $L^{2}$-error estimate for the velocity). Suppose that the continuous problem satisfies the elliptic regularity condition

$$
\sqrt{\mu}\|\boldsymbol{u}\|_{H^{2}(\Omega)}+\frac{1}{\sqrt{\mu}}\|\boldsymbol{\sigma}\|_{H^{1}(\Omega)}+\frac{1}{\sqrt{\mu}}\|p\|_{H^{1}(\Omega)} \leq \frac{C}{\sqrt{\mu}}\|\boldsymbol{f}\| .
$$

Copyright $@$ ㅇ by SIAM. Unauthorized reproduction of this article is prohibited. 
Then

$$
\sqrt{\mu}\left\|\boldsymbol{u}-\boldsymbol{u}_{h}\right\| \leq C h\left(\sqrt{\mu}\left\|\boldsymbol{u}-\boldsymbol{u}_{h}\right\|_{H^{1}(\Omega)}+\frac{1}{\sqrt{\mu}}\left\|\boldsymbol{\sigma}-\boldsymbol{\sigma}_{h}\right\|+\frac{1}{\sqrt{\mu}}\left\|p-p_{h}\right\|\right) .
$$

Proof. Let $(\boldsymbol{\omega}, \pi, \boldsymbol{S}) \in \mathcal{X}$ be the solution of the following adjoint problem:

$$
\begin{aligned}
\nabla \cdot \boldsymbol{S}-\nabla \pi & =\frac{\mu}{\ell^{2}}\left(\boldsymbol{u}-\boldsymbol{u}_{h}\right) & & \text { in } \Omega, \\
-\nabla \cdot \boldsymbol{\omega} & =0 & & \text { in } \Omega, \\
\frac{1}{2 \mu} \boldsymbol{S}+\nabla^{S} \boldsymbol{\omega} & =\mathbf{0} & & \text { in } \Omega,
\end{aligned}
$$

with $\boldsymbol{\omega}=\mathbf{0}$ on $\partial \Omega$ and where $\ell$ is a characteristic length scale of the problem that has been introduced to keep the dimensionality, but that will play no role in the final result. Let also $\left(\tilde{\boldsymbol{\omega}}_{h}, \tilde{\pi}_{h}, \tilde{\boldsymbol{S}}_{h}\right)$ be the best approximation to $(\boldsymbol{\omega}, \pi, \boldsymbol{S})$ in $\mathcal{X}_{h}$. Testing (4.27) with $\boldsymbol{u}-\boldsymbol{u}_{h}$, (4.28) with $p-p_{h}$, and (4.29) with $\boldsymbol{\sigma}-\boldsymbol{\sigma}_{h}$, we immediately obtain

$$
\begin{aligned}
\frac{\mu}{\ell^{2}}\left\|\boldsymbol{u}-\boldsymbol{u}_{h}\right\|^{2} & =B\left(\left(\boldsymbol{u}-\boldsymbol{u}_{h}, p-p_{h}, \boldsymbol{\sigma}-\boldsymbol{\sigma}_{h}\right),(\boldsymbol{\omega}, \pi, \boldsymbol{S})\right) \\
& =B_{\mathrm{stab}}\left(\left(\boldsymbol{u}-\boldsymbol{u}_{h}, p-p_{h}, \boldsymbol{\sigma}-\boldsymbol{\sigma}_{h}\right),(\boldsymbol{\omega}, \pi, \boldsymbol{S})\right) \\
& -\alpha_{\sigma} 2 \mu \sum_{K}\left\langle P_{\sigma}^{\perp}\left(\frac{1}{2 \mu} \boldsymbol{S}+\nabla^{S} \boldsymbol{\omega}\right), P_{\sigma}^{\perp}\left(\nabla^{S}\left(\boldsymbol{u}-\boldsymbol{u}_{h}\right)\right)\right\rangle_{K} \\
& -\alpha_{p} 2 \mu \sum_{K}\left\langle P_{\sigma}^{\perp}(\nabla \cdot \boldsymbol{\omega}), P_{\sigma}^{\perp}\left(\nabla \cdot\left(\boldsymbol{u}-\boldsymbol{u}_{h}\right)\right)\right\rangle_{K} \\
& -\alpha_{u} \frac{h^{2}}{\mu} \sum_{K}\left\langle P_{u}^{\perp}(\nabla \pi-\nabla \cdot \boldsymbol{S}), P_{u}^{\perp}\left(\nabla\left(p-p_{h}\right)-\nabla \cdot\left(\boldsymbol{\sigma}-\boldsymbol{\sigma}_{h}\right)\right)\right\rangle_{K} \\
& -\delta_{0} \frac{h}{2 \mu} \sum_{E}\left\langle\llbracket \boldsymbol{n} \pi-\boldsymbol{n} \cdot \boldsymbol{S} \rrbracket, \llbracket \boldsymbol{n}\left(p-p_{h}\right)-\boldsymbol{n} \cdot\left(\boldsymbol{\sigma}-\boldsymbol{\sigma}_{h}\right) \rrbracket\right\rangle_{E},
\end{aligned}
$$

where we have made use of the definition (3.19) of $B_{\mathrm{stab}}$. Note that we have included $\boldsymbol{S}$ in $P_{\sigma}^{\perp}\left(\frac{1}{2 \mu} \boldsymbol{S}+\nabla^{S} \boldsymbol{\omega}\right)$ because it does not affect the definition of $B_{\text {stab }}$ when applied to discrete finite element functions.

The second and third terms in the right-hand side of (4.30) are zero because of (4.29) and (4.28), respectively, and the last one is also zero because of the weak continuity of the stresses associated to problems (4.27)-(4.29). Therefore, only the first and fourth terms need to be bounded.

Using Lemma 4.2, for the first term in (4.30) we have

$$
\begin{aligned}
& B_{\text {stab }}\left(\left(\boldsymbol{u}-\boldsymbol{u}_{h}, p-p_{h}, \boldsymbol{\sigma}-\boldsymbol{\sigma}_{h}\right),(\boldsymbol{\omega}, \pi, \boldsymbol{S})\right) \\
& \quad=B_{\text {stab }}\left(\left(\boldsymbol{u}-\boldsymbol{u}_{h}, p-p_{h}, \boldsymbol{\sigma}-\boldsymbol{\sigma}_{h}\right),\left(\boldsymbol{\omega}-\tilde{\boldsymbol{\omega}}_{h}, \pi-\tilde{\pi}_{h}, \boldsymbol{S}-\tilde{\boldsymbol{S}}_{h}\right)\right) .
\end{aligned}
$$

Using the interpolation properties and the shift assumption (4.25) it follows that

$$
\begin{aligned}
& \left\|\boldsymbol{\omega}-\tilde{\boldsymbol{\omega}}_{h}\right\|_{H^{1}(\Omega)} \leq C h\|\boldsymbol{\omega}\|_{H^{2}(\Omega)} \leq C h \frac{1}{\ell^{2}}\left\|\boldsymbol{u}-\boldsymbol{u}_{h}\right\|, \\
& \left\|\boldsymbol{S}-\tilde{\boldsymbol{S}}_{h}\right\| \leq C h\|\boldsymbol{S}\|_{H^{1}(\Omega)} \leq C h \frac{\mu}{\ell^{2}}\left\|\boldsymbol{u}-\boldsymbol{u}_{h}\right\|, \\
& \left\|\pi-\tilde{\pi}_{h}\right\| \leq C h\|\pi\|_{H^{1}(\Omega)} \leq C h \frac{\mu}{\ell^{2}}\left\|\boldsymbol{u}-\boldsymbol{u}_{h}\right\| .
\end{aligned}
$$

Copyright $\odot$ by SIAM. Unauthorized reproduction of this article is prohibited. 
From these expressions it can be easily checked that (4.31) can be bounded by

$$
\begin{aligned}
& B_{\mathrm{stab}}\left(\left(\boldsymbol{u}-\boldsymbol{u}_{h}, p-p_{h}, \boldsymbol{\sigma}-\boldsymbol{\sigma}_{h}\right),(\boldsymbol{\omega}, \pi, \boldsymbol{S})\right) \\
& \quad \leq C h \frac{\sqrt{\mu}}{\ell^{2}}\left\|\boldsymbol{u}-\boldsymbol{u}_{h}\right\|\left(\sqrt{\mu}\left\|\boldsymbol{u}-\boldsymbol{u}_{h}\right\|_{H^{1}(\Omega)}+\frac{1}{\sqrt{\mu}}\left\|\boldsymbol{\sigma}-\boldsymbol{\sigma}_{h}\right\|+\frac{1}{\sqrt{\mu}}\left\|p-p_{h}\right\|\right) .
\end{aligned}
$$

Let us check this bound for example for the term in $B_{\text {stab }}\left(\left(\boldsymbol{u}-\boldsymbol{u}_{h}, p-p_{h}, \boldsymbol{\sigma}-\right.\right.$ $\left.\left.\boldsymbol{\sigma}_{h}\right),(\boldsymbol{\omega}, \pi, \boldsymbol{S})\right)$ involving boundary integrals, for which we have

$$
\begin{aligned}
\delta_{0} & \frac{h}{\mu} \sum_{E}\left\langle\llbracket \boldsymbol{n}\left(\tilde{\pi}_{h}-\pi\right)-\boldsymbol{n} \cdot\left(\tilde{\boldsymbol{S}}_{h}-\boldsymbol{S}\right) \rrbracket, \llbracket \boldsymbol{n}\left(p_{h}-p\right)-\boldsymbol{n} \cdot\left(\boldsymbol{\sigma}_{h}-\boldsymbol{\sigma}\right) \rrbracket\right\rangle_{E} \\
& \leq C \frac{h}{\mu}\left[h^{-1 / 2}\left(\left\|\tilde{\pi}_{h}-\pi\right\|+\left\|\tilde{\boldsymbol{S}}_{h}-\boldsymbol{S}\right\|\right)+h^{1 / 2}\left(\left\|\tilde{\pi}_{h}-\pi\right\|_{H^{1}(\Omega)}+\left\|\tilde{\boldsymbol{S}}_{h}-\boldsymbol{S}\right\|_{H^{1}(\Omega)}\right)\right] \\
& \times\left[h^{-1 / 2}\left(\left\|p_{h}-p\right\|+\left\|\boldsymbol{\sigma}_{h}-\boldsymbol{\sigma}\right\|\right)+h^{1 / 2}\left(\left\|p_{h}-p\right\|_{H^{1}(\Omega)}+\left\|\boldsymbol{\sigma}_{h}-\boldsymbol{\sigma}\right\|_{H^{1}(\Omega)}\right)\right] \\
& \leq C \frac{h}{\ell^{2}}\left\|\boldsymbol{u}-\boldsymbol{u}_{h}\right\|\left(\left\|p-p_{h}\right\|+\left\|\boldsymbol{\sigma}-\boldsymbol{\sigma}_{h}\right\|\right) .
\end{aligned}
$$

The rest of the terms in $B_{\text {stab }}\left(\left(\boldsymbol{u}-\boldsymbol{u}_{h}, p-p_{h}, \boldsymbol{\sigma}-\boldsymbol{\sigma}_{h}\right),(\boldsymbol{\omega}, \pi, \boldsymbol{S})\right)$ can be bounded similarly. We omit the details.

It only remains to bound the fourth term in (4.30). This is again easily done using that $\|\boldsymbol{S}\|_{H^{1}(\Omega)}+\|\pi\|_{H^{1}(\Omega)} \leq C \frac{\mu}{\ell^{2}}\left\|\boldsymbol{u}-\boldsymbol{u}_{h}\right\|$, which yields

$$
\begin{gathered}
\alpha_{u} \frac{h^{2}}{\mu} \sum_{K}\left\langle P_{u}^{\perp}(\nabla \pi-\nabla \cdot \boldsymbol{S}), P_{u}^{\perp}\left(\nabla\left(p-p_{h}\right)-\nabla \cdot\left(\boldsymbol{\sigma}-\boldsymbol{\sigma}_{h}\right)\right)\right\rangle_{K} \\
\leq C \frac{h^{2}}{\mu} \frac{\mu}{\ell^{2}}\left\|\boldsymbol{u}-\boldsymbol{u}_{h}\right\|\left(\left\|\nabla p-\nabla p_{h}\right\|+\left\|\nabla \cdot \boldsymbol{\sigma}-\nabla \cdot \boldsymbol{\sigma}_{h}\right\|\right) .
\end{gathered}
$$

Using this and (4.32) in (4.30) the theorem follows.

5. Concluding remarks. Let us conclude with some remarks concerning the numerical formulation presented in this paper. This formulation is an application of subgrid scale concept to the stress-displacement-pressure formulation of the Stokes problem. Apart from the novelty of this application, a feature of the formulation is to consider the spaces of subgrid scales orthogonal to the finite element spaces. Other ingredients original of this paper are the basis for the design of the parameters of formulation and the introduction of subgrid scales on the element boundaries.

From the point of view of the numerical analysis, the method presented is stable and optimally accurate using arbitrary interpolations for the displacement, the pressure and the stresses. Comparing it with the Galerkin method using stable interpolations, exactly the same regularity requirements are needed and the same convergence rates are obtained, also in the same norms. Therefore, the main goal has been achieved.

The accuracy of the method obtained in some numerical experiments is the one expected from the convergence analysis. Theoretical convergence rates are exactly recovered. We have preferred to skip the results of numerical testing in the linear setting analyzed in this paper and to postpone them for a more extensive numerical experimentation in more complex applications.

The practical interest of the problem studied is obvious. As it has been mentioned in the Introduction, this is nothing but a model for more complex situations. Typically, viscoelastic flows are often posed as an example of a problem that requires the 
interpolation of the stresses, but this can also be done for nonlinear models such as damage or plasticity in solid mechanics, and non-Newtonian fluids or even turbulence models in fluid mechanics. When designing an extension of the formulations presented here to these more complex situations, the most important idea to bear in mind is which is the stabilization mechanism introduced by the formulations proposed. The analysis dictates that pressure is stabilized by the term proportional to $P_{u}^{\perp}\left(\nabla p_{h}\right)$ introduced in the continuity equation, and the displacement gradient is stabilized by the term proportional to $P_{\sigma}^{\perp}\left(\nabla^{S} \boldsymbol{u}_{h}\right)$ introduced in the momentum equation. This is the essential point. The only condition on the factors that multiply these terms is that they have to yield an adequate scaling and order of convergence.

\section{REFERENCES}

[1] D.N. Arnold, G. Awanou, and R. Winther, Finite elements for symmetric tensors in three dimensions, Math. Comp., 77 (2008), pp. 1229-1251.

[2] D.N. Arnold and R. Winther, Mixed finite elements for elasticity, Numer. Math., 92 (2002), pp. 401-419.

[3] F.P.T. BaAijens, M.A. Hulsen, and P.D. Anderson, The use of mixed finite element methods for viscoelastic fluid flow analysis, Chapter 14 in Encyclopedia of Computational Mechanics, E. Stein, R. de Borst, and T.J.R. Hughes, eds., John Wiley \& Sons, New York, 2004, pp. 481-498.

[4] R. Becker and M. BraAck, A finite element pressure gradient stabilization for the Stokes equations based on local projections, Calcolo, 38 (2001), pp. 173-199.

[5] M. Behr, L.P. Franca, And T.E. Tezduyar, Stabilized finite element methods for the velocitypressure-stress formulation of incompressible flows, Comput. Methods Appl. Mech. Engrg., 104 (1993), pp. 31-48.

[6] J. Bonvin, M. Picasso, And R. Stenberg, GLS and EVSS methods for a three fields Stokes problems arising from viscoelastic flows, Comput. Methods Appl. Mech. Engrg., 190 (2001), pp. 3893-3914.

[7] M. BraAcK And E. Burman, Local projection stabilization for the Oseen problem and its interpretation as a variational multiscale method, SIAM J. Numer. Anal., 43 (2006), pp. 25442566.

[8] F. Brezzi and M. Fortin, Mixed and Hybrid Finite Element Methods, Springer-Verlag, New York, 1991.

[9] R. CodinA, Stabilization of incompressibility and convection through orthogonal sub-scales in finite element methods, Comput. Methods Appl. Mech. Engrg., 190 (2000), pp. 1579-1599.

[10] R. CodinA, Stabilized finite element approximation of transient incompressible flows using orthogonal subscales, Comput. Methods Appl. Mech. Engrg., 191 (2002), pp. 4295-4321.

[11] R. Codina, Analysis of a stabilized finite element approximation of the Oseen equations using orthogonal subscales, Appl. Numer. Math., 58 (2008), pp. 264-283.

[12] R. Codina, Finite element approximation of the hyperbolic wave equation in mixed form, Comput. Methods Appl. Mech. Engrg., 197 (2008), pp. 1305-1322.

[13] C.R. Dohrmann And P.B. Bochev, A stabilized finite element method for the Stokes problem based on polynomial pressure projections, Int. J. Num. Meth. Fluids, 46 (2004), pp. 183201.

[14] M. Fortin, R. GuÉnette, And R. Pierre, Numerical analysis of the modified EVSS method, Comput. Methods Appl. Mech. Engrg., 143 (1997), pp. 79-95.

[15] M. Fortin And R. Pierre, On the convergence of the mixed method of crochet and marchal for viscoelastic flows, Comput. Methods Appl. Mech. Engrg., 73 (1989), pp. 341-350.

[16] L. Franca And R. Stenberg, Error analysis of some Galerkin least-squares methods for the elasticity equations, SIAM J. Numer. Anal., 28 (1991), pp. 1680-1697.

[17] G.N. Gatica, Analysis of a new augmented mixed finite element method for linear elasticity allowing $R T_{0}-P_{1}-P_{0}$ approximations, ESAIM: Mathematical Modelling and Numerical Analysis, 40 (2006), pp. 1-28.

[18] G.N. Gatica, A. Márquez, and S. Meddahi, An augmented mixed finite element method of low cost for three-dimensional linear elasticity problems, preprint 07-21, Departamento de Ingenieria Matematica, Universidad de Concepcion, 2007.

[19] R. GuÉnette And M. Fortin, A new mixed finite element method for computing viscoelastic flows, J. Non-Newtonian Fluid Mechanics, 60 (1995), pp. 27-52. 
[20] T.J.R. Hughes, Multiscale phenomena: Green's function, the Dirichlet-to-Neumann formulation, subgrid scale models, bubbles and the origins of stabilized formulations, Comput. Methods Appl. Mech. Engrg., 127 (1995), pp. 387-401.

[21] T.J.R. Hughes, G.R. Feisóo, L. Mazzei, And J.B. Quincy, The variational multiscale method-a paradigm for computational mechanics, Comput. Methods Appl. Mech. Engrg., 166 (1998), pp. 3-24.

[22] P. KNobloch AND L. Tobiska, On Korn's first inequality for quadrilateral nonconforming finite elements of first order approximation properties, Int. J. Numer. Anal. Modeling, 2 (2005), pp. 439-458.

[23] J.M. Marchal and M.J. Crochet, A new mixed finite-element for calculating viscoelastic flow, J. Non-Newtonian Fluid Mechanics, 26 (1987), pp. 77-114.

[24] G. Matthies, P. Skrzypacz, and L. Tobiska, A unified convergence analysis for local projection stabilisations applied to the Oseen problem, Math. Modelling Numer. Anal., 41 (2007), pp. $713-742$.

[25] V. RUAS, Une méthode mixte contrainte-déplacement-pression pour la résolution de problèmes de viscoélasticité incompressible en déformations planes, Comptes Rendus de l'Académie des Sciences. Série 2, 301 (1985), pp. 1171-1174.

[26] V. RuAS, Finite element methods for the three-field Stokes system, RAIRO Modélisation Mathématique et Analyse Numérique, 30 (1996), pp. 489-525.

[27] V. RuAs, Galerkin-least-squares finite element methods for the three-field Stokes system in $\mathbb{R}^{3}$, Comput. Methods Appl. Mech. Engrg., 142 (1997), pp. 235-256.

[28] D. SANDRI, Analysis of a three-fields approximation of the Stokes problem, RAIRO Modélisation Mathématique et Analyse Numérique, 23 (1993), pp. 817-841.

Copyright (c) by SIAM. Unauthorized reproduction of this article is prohibited. 